\title{
Wie stark verdrängen oder ergänzen sich (neue und alte) Medien?
}

\author{
Wolfgang Seufert / Claudia Wilhelm
}

Der Beitrag präsentiert empirische Ergebnisse zur Stärke substitutiver Beziehungen zwischen Mediengattungen auf Basis der Schätzung von Strukturgleichungsmodellen, deren Datengrundlage Zeitverwendungsdaten aus der ARD/ZDF-Langzeitstudie Massenkommunikation der Erbebungsjabre 1995, 2005 und 2010 sind. Theoretische Grundlage ist ein mikroökonomisch fundiertes Modell zur Erklärung medienübergreifender Entscheidungen der Medienwabl. Es werden langfristig wirksame Substitutionsbeziehungen zwischen alten und neuen Medien, die auf einer Veränderung der Präferenzordnung für Mediennutzungs-und nichtmedialen Aktivitäten nach einer Medieninnovation beruben, von situativ bedingten kurzfristigen Substitutionsbeziebungen unterschieden. Diese werden darin sichtbar, dass eine höbere Nutzungsdauer einer Mediengattung systematisch mit einer niedrigeren Nutzungsdauer einer anderen Mediengattung einbergebt. Die Schätzergebnisse zeigen, dass es zwischen der Nutzung des Internets und der Nutzung aller anderen aktuellen Medien sowobl langfristige Substitutionseffekte als auch kurzfristige Substitutionsbeziehungen gibt. Sie sind aber sebr schwach und durchwegs niedriger als zu nichtmedialen Freizeitaktivitäten. Belege für eine komplementäre „Themore-the-more"-Nutzung, bei der eine höbere Nutzungsdauer des Internets dauerhaft mit einer höheren Nutzungsdauer anderer aktueller Medien einhergebt, finden sich nicht.

Schlagwörter: Mediennutzung, Zeitverwendung, Mediensubstitution, Komplementarität

\section{Bestimmung der Stärke von Substitutionsbeziehungen zwischen Mediengattungen auf Grundlage eines Zeitallokationsmodells der Mediennutzung}

Mit der Verbreitung eines „neuen Mediums“ wird immer wieder die Frage gestellt, ob dessen Nutzung zu Lasten der „alten Medien“ gehen wird (Langenbucher 1968; Lerg 1981; Kiefer 1989; Dimmick 2003; Mögerle 2009). Die Stärke solcher Substitutionseffekte ist nicht nur aufgrund der damit verbundenen medienwirtschaftlichen Folgen von Interesse ${ }^{1}$ sondern auch wegen möglicher Verschiebungen der Relevanz einzelner Mediengattungen für die öffentliche Meinungsbildung. ${ }^{2}$ Seit Mitte der 1990er Jahre stehen deshalb vor allem die Auswirkungen des Internets bzw. des WWW-Angebotes auf die Nutzung der aktuellen Medien Fernsehen, Hörfunk und Tageszeitung verstärkt im Fokus kommunikationswissenschaftlicher und medienökonomischer Forschung (u. a. Bromley/Bowles 1995; Hagen 1998; Waldfogel 2002; Scherer/Schlütz 2004; de Waal et al. 2005; Mögerle 2009).

1 In den 1960er Jahren wurde das neue Medium Fernsehen als so bedrohlich für die Existenz der etablierten Massenmedien Presse und Filme empfunden, dass sich sogar zwei Bundestags-Kommissionen - nach ihren Vorsitzenden Michel-Kommission (Deutscher Bundestag 1967) und Günther-Kommission (Deutscher Bundestag 1968) genannt - mit dem intermedialen Wettbewerb beschäftigten.

2 Die FCC hat 2003 die Reduzierung ihrer Schranken für crossmediale Konzentration bei den traditionellen Medien mit der inhaltlichen Vielfalt im Internet begründet (vgl. Markus 2007). 
Um die Frage nach dem Substitutionspotenzial eines neuen Mediums beantworten zu können, bedarf es eines Erklärungsmodells für das Auswahlverhalten von Rezipienten zwischen unterschiedlichen Medienangeboten. Kommunikationswissenschaftliche Studien stehen dabei zum großen Teil in der Tradition des Uses \& Gratifications-Ansatzes (U\&G). Man geht davon aus, dass die Medienselektion primär aufgrund erwarteter Gratifikationen hinsichtlich der Befriedigung von spezifischen Orientierungs-, Kommunikations- und Unterhaltungsbedürfnissen erfolgt, wobei sich diese Erwartungen aufgrund von Erfahrungen mit den bislang erhaltenen Gratifikationen herausbilden (Blumler/Katz 1974; Rosengren et al. 1985). Die Wahrscheinlichkeit, dass die zusätzliche Nutzung der Angebote einer neuen Mediengattung zu einer geringeren Nutzung der Angebote einer existierenden Mediengattung führt, steigt danach, a) je stärker sich die beiden Gratifikationsspektren überschneiden und b) je größer die Zahl der Gratifikationsdimensionen ist, auf denen das neue Medium aus der Sicht der Rezipienten bessere Leistungen erbringt als das alte. Da neue Mediengattungen aber in der Regel nicht auf allen Gratifikationsdimensionen überlegen sind, geht die Kommunikationswissenschaft überwiegend davon aus, dass ein „Gesetz von der Komplementarität“3 gilt, wonach die völlige Verdrängung eines alten Mediums durch ein neues nicht zu erwarten ist (Kiefer 1989: 348). Wirtschaftliche und medienpolitische Konsequenzen ergeben sich allerdings nicht erst bei einer vollständigen, sondern bereits bei einer umfangreicheren partiellen Substitution. ${ }^{4}$

Das „neue Medium“ Internet hat aufgrund seines sehr breiten Gratifikationsspektrums ein solches Potenzial. So gibt es sowohl Studien, die eine Überlegenheit im Bereich der Informationsleistungen und hierbei vor allem bei der Breite des Angebots und der zeitlichen Unabhängigkeit der Nutzung von aktuellen Nachrichten ${ }^{5}$ feststellen (u. a. Dimmick et al. 2004), als auch Studien, die auch im Bereich einzelner Unterhaltungs-

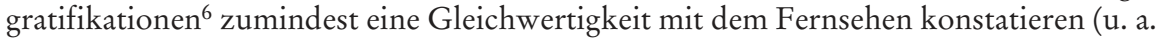
Scherer/Schlütz 2004).

Die Ergebnisse aus Umfragen zur subjektiven Bewertung von Mediengratifikationen können Substitutionseffekte erklären, sie lassen aber allein noch keine Aussagen über die Auswirkungen auf das tatsächliche Nutzungsverhalten zu. Hierzu werden sie deshalb in der Regel mit Daten zum Mediennutzungsverhalten verknüpft. Beispielsweise werden Selbstauskünfte über eine geringere oder stärkere Nutzung alter Medien nach

3 Die deutsche Kommunikationswissenschaft nimmt dabei oft auf das vor 100 Jahren von Riepl formulierte Gesetz Bezug, wonach seit dem Altertum trotz der Entwicklung neuer Techniken der Nachrichtenübertragung sämtliche alte Formen (wenn auch in geringem Umfang) weiter genutzt würden (Riepl 1987). Es wurde als „Gesetz von der Komplementarität“ auch auf Massenmedien übertragen (Langenbucher 1968). Allerdings wird zu Recht kritisiert, dass es empirisch wenig gehaltvoll ist, da streng genommen bereits ein einzelner Nutzer irgendwo auf der Welt ausreicht, um die Weiterexistenz eines Mediums zu belegen (Peiser 2002).

4 Der von Lerg (1981: 200) für eine partielle Ersetzung vorgeschlagene Begriff der Kompensation hat sich in der Kommunikationswissenschaft nicht durchsetzen können.

5 Dimmick et al. (2004: 28) gehen von einer eignen Gratifikationsdimension „Nutzungspotenzial“" (gratification opportunities) neben Informations-, Kommunikations- und Unterhaltungsgratifikationen aus, die durch acht Befragungs-Items abgefragt werden kann. Sie stellen eine Überlegenheit des Internets gegenüber Nachrichtenangeboten im terrestrischen Fernsehen, im Radio und in der Zeitung fest, nicht aber gegenüber den Nachrichtenkanälen im Kabel-TV.

6 Scherer/Schlütz (2004: 17) vergleichen das Gratifikationsspektrum von WWW und Fernsehen auf Basis von zehn Befragungsitems, die zu fünf Gratifikationsdimensionen geordnet werden. Eine Gleichwertigkeit gibt es bei den erhaltenen Gratifikationen für die Dimension Unterhaltung, nicht aber für die Dimension Entspannung. 
dem Beginn der Internetnutzung (u. a. Dimmick et al. 2004) herangezogen, oder es werden auf Basis einer Sekundärauswertung repräsentativer Nutzerbefragungen Vergleiche der Nutzungshäufigkeiten oder Nutzungsdauern von Internetnutzern und Nichtnutzern durchgeführt (u. a. Hagen 1998). Allerdings erlaubt dieses Vorgehen meist nur qualitative Tendenzaussagen, wonach einzelne Mediengattungen stärker oder weniger stark von Substitutionseffekten betroffen sind. Quantitative Aussagen, wonach die zusätzliche Nutzung eines neuen Mediums um x Prozent zu einer Reduzierung der Nutzung eines alten Mediums um y Prozent führt, sind in kommunikationswissenschaftlichen und medienökonomischen Studien seltener zu finden (u. a. Waldfogel 2002; Salwen et al. 2004; de Waal et al. 2005; Kollo/Meyer-Lucht 2007).

Auch der folgende Beitrag möchte eine solche Quantifizierung der relativen Stärke von Substitutionsbeziehungen zwischen Mediengattungen vornehmen. Sie basiert auf Schätzungen von Strukturgleichungsmodellen, deren theoretische Grundlage nicht der U\&G-Ansatz zur Medienauswahl ist, sondern eine Übertragung der mikroökonomischen Theorie der Konsumwahl (u. a. Varian 2007: 31f.) auf Medienauswahlentscheidungen. An die Stelle des Modells zur Erklärung der Aufteilung eines aus Geldeinheiten bestehenden Konsumbudgets tritt ein analoges Modell zur Erklärung der Allokation individuell verfügbarer Zeitbudgets auf unterschiedliche mediale Nutzungsaktivitäten und nichtmediale Aktivitäten (Seufert/Ehrenberg 2007: 24f.). Datengrundlage für die vorgestellten Modellschätzungen sind die Erhebungen der ARD/ZDF-Langzeitstudie Massenkommunikation (MK) aus den Jahren 1995, 2005 und 2010 (zur Methodik vgl. Reitze/Ridder 2011).

Gegenüber dem in der Kommunikationswissenschaft vorherrschenden Konzept des U\&G-Ansatzes ergeben sich hierdurch drei wesentliche Unterschiede, auf die im Rahmen des Beitrages noch genauer eingegangen werden wird:

1. Es werden langfristig wirksame Substitutionseffekte durch die Verbreitung neuer Medien von situativ bedingten kurzfristigen Substitutionsbeziehungen zwischen Mediengattungen unterschieden. Erstere sind eine Folge von veränderten Präferenzordnungen, d. h. einer veränderten Rangfolge der subjektiven Wertschätzungen für einzelne Mediennutzungsaktivitäten. Letztere sind zu jedem Zeitpunkt latent vorhanden und zeigen sich, wenn sich Handlungsrestriktionen kurzfristig verändern entweder hinsichtlich der relativen Kosten einzelner Aktivitäten oder hinsichtlich des insgesamt verfügbaren Zeitbudgets.

2. Substitutionsbeziehungen kann es auch zwischen Mediennutzungsaktivitäten geben, die keinen gemeinsamen spezifischen Nutzen (keine übereinstimmenden Gratifikationsdimensionen) haben. Dies impliziert, dass es immer auch Substitutionsbeziehungen zwischen Mediennutzungsaktivitäten und einer Fülle nichtmedialer Aktivitäten gibt.

3. Eine methodische Konsequenz des mikroökonomischen Ansatzes ist, dass man Präferenzordnungen, d. h. die subjektiven Wertschätzungen für einzelne Konsumgüter oder Aktivitäten, besser indirekt aus beobachtbaren Nachfragemengen bzw. Aktivitätsdanern erschließen sollte, als sie direkt abzufragen (Konzept der bekundeten Präferenzen). Insbesondere liefern Angaben zur Häufigkeit einer Aktivität (bzw. von Kaufakten) keine ausreichenden Informationen, da eine regelmäßige (täglich oder mehrmals täglich ausgeführte) Mediennutzungsaktivität sowohl von kurzer als auch von langer Dauer sein kann (vgl. Meulemann 2012: 252f.).

Bei allen präsentierten Ergebnissen ist zu berücksichtigen, dass die bisherige Erfassung der „Mediengattung Internet“ im MK-Datensatz methodisch nicht unproblematisch ist: Zum einen stellt sich die Frage, welcher Anteil der Internet-Kommunikation eigentlich 
eine Mediennutzungsaktivität darstellt. Zum anderen bleibt unklar, welche Medieninhalte im Internet wirklich neuartig sind und welche Inhalte nur ein neuer Vertriebsweg für die Angebote „alter Medien“ sind:

- Das Internet ermöglicht eine Vielzahl von Aktivitäten, die vor der Verlagerung ins Internet zur privaten Individualkommunikation (z. B. E-Mail, soziale Netzwerke) oder zur Wirtschaftskommunikation (z. B. E-Commerce, E-Banking) gerechnet worden wären. Nach den Erhebungen der ARD/ZDF-Onlinestudien machen diese Anwendungen den größten Teil aller Internetaktivitäten aus (van Eimeren/Frees 2012: 369). Internetnutzung ist insofern im traditionellen Verständnis nur zu einem Bruchteil die Nutzung von Medieninhalten.

- Da mit der Internettechnologie sämtliche „alten“ Medieninhalte in digitalisierter Form verbreitet werden können, ergibt sich hier eine Abgrenzungs- und damit Messproblematik zu allen anderen Mediengattungen. Ist die Rezeption des Audio-Streams eines Radioprogrammes noch Radionutzung oder schon Internetnutzung? Oder ist die Lesezeit für ein E-Book noch der Mediengattung Buch zuzurechnen oder ebenfalls schon dem Internet? Wenn man beide Fragen zugunsten der Kategorie Internetnutzung beantwortet, wird das Potenzial für Substitutionseffekte zwischen der Internetnutzung und der Nutzung aller alten aktuellen wie nichtaktuellen Medien natürlich sehr groß. Beantwortet man beide Fragen zugunsten der herkömmlichen Medienkategorien, bleiben als spezifisches mediales Angebot der Mediengattung Internet letztlich nur die interaktiv nutzbaren und gleichzeitig verschiedene Medienformen integrierenden Websites übrig, die in den 1990er Jahren als „Online-Multimedia" bezeichnet wurden.

\section{Mediennutzung als Zeitallokation}

Da mikroökonomisch fundierte Ansätze der Medienauswahl ${ }^{7}$ in der Kommunikationswissenschaft wenig rezipiert wurden, soll vor der Präsentation der empirischen Ergebnisse zunächst kurz das theoretische Modell der Konsumbudgetallokation und seine Übertragung auf die Zeitverwendung erläutert werden.

\subsection{Das mikroökonomische Modell der Nachfrage nach Konsumgütern}

Konsumenten unterscheiden sich sowohl darin, welche Waren und Dienstleistungen sie überhaupt aus einem vorhandenen Konsumgüterangebot auswählen, als auch in den von ihnen jeweils nachgefragten Gütermengen. Die mikroökonomische Konsumtheorie will erklären, welche Einflussfaktoren zu diesen unterschiedlichen Konsumentenentscheidungen führen.

Als eine Ursache werden die individuell unterschiedlichen Präferenzordnungen angesehen. Man geht davon aus, dass sich die Nachfrage nach einem Konsumgut nicht aus objektiven Produkteigenschaften ableitet, sondern aus subjektiven Nutzenbewertungen. Eine zentrale Annahme der mikroökonomischen Konsumtheorie ist dabei das Gesetz vom abnebmenden Grenznutzen aller Konsumgüter: Der subjektive Nutzen einer weiteren Einheit ist in der Regel geringer, als der Nutzen der jeweils zuvor konsumierten

7 Insbesondere in den USA hat sich eine mikroökonomisch fundierte Tradition der Erklärung des Medienselektionsverhaltens entwickelt. Versucht wird damit jeweils, die inhaltliche Ausdifferenzierung des Medienangebotes als Wechselbeziehung der Auswahlentscheidungen von Rezipienten zwischen verschiedenen Medienangeboten und den dadurch ausgelösten Anbieterreaktionen zu erklären (u. a. Steiner 1952; Owen et al. 1974; Owen/Wildmann 1992, Waterman 2006). 
Einheit. Diese Sättigungseffekte sind ebenfalls individuell unterschiedlich und sie treten bei unterschiedlichen Konsumgütern unterschiedlich schnell auf. Dies bedeutet, dass sich aus Sicht eines einzelnen Konsumenten der gleiche Gesamtnutzen von zwei (oder mehr) Konsumgütern durch unterschiedliche Kombinationen von Gütermengen erreichen lässt. Alle Güter mit einem subjektiv positiven Nutzen sind damit prinzipiell austauschbar. Unterschiedlich ist lediglich der jeweilige Grad dieser Substituierbarkeit.

Welche Güterkombination ein Konsument tatsächlich nachfragt, hängt nicht nur von seiner durch Persönlichkeitseigenschaften, Erziehung oder Lebenserfahrungen geprägten Präferenzordnung ${ }^{8}$ ab, sondern auch von zwei weiteren Faktoren, die kurzfristigen Schwankungen unterliegen können: zum einen von der Höhe des für den Konsum verwendbaren Teils des Einkommens (das Konsumbudget) und zum anderen von den relativen Kosten der angebotenen Güter (die Konsumgüterpreise). Beide Faktoren bilden zusammen die Handlungsrestriktionen des einzelnen Konsumenten, indem sie die Grenze für diejenigen Güterbündel festlegen, die mit dem verfügbaren Geld überhaupt gekauft werden können.

Die mikroökonomische Konsumtheorie geht nun davon aus, dass sich ein rational handelnder Konsument nutzenmaximierend verhalten wird und Konsumgüter in derjenigen Mengenkombination kauft, mit der er sein subjektiv höchstes Gesamtnutzenniveau erreichen kann. Gegen die Anwendung dieses mikroökonomischen Konsummodells bzw. anderer Rational-Choice-Ansätze auf die Mediennutzung wird häufig der Einwand vorgebracht, dass es wegen der in der Regel geringen Preise (oder wie im Fall des Free-TV einem Preis von Null) systematisch zu irrationalen oder zumindest unbewussten Entscheidungen komme (Jäckel 1992).

Diese Argumentation verwechselt jedoch Irrationalität mit begrenzt rationalem Verhalten aufgrund unvollkommener Marktübersicht, das auch mit einem gewohnheitsmäßigen unbewussten Verhalten vereinbar ist. Irrationalität im ökonomischen Sinn läge vor, wenn ein Konsument permanent einen Teil seines Budgets für ein Konsumgut verwendet, das ihm weniger Nutzen bringt, als es andere Güter mit dem gleichen Preis könnten (vgl. Becker 1962: 185). Ein solches Verhalten würde aber voraussetzen, dass Konsumenten die Qualität bzw. den Nutzen von Produkten generell nicht richtig beurteilen können und damit auch nicht in der Lage sind, ihre Erfahrungen in künftige Auswahlentscheidungen einfließen zu lassen.

Das Argument der Niedrigkostensituation unterstellt deshalb im Kern nicht Irrationalität, sondern richtet sich vielmehr gegen die Annahme einer vollkommen rationalen Konsumwahl, bei der sich der Konsument jedes Mal aktiv einen Überblick über alle vorhandenen Angebote (und deren Preise) verschafft. Da damit jedoch Informationsund Suchkosten (Zeit- und/oder Geldkosten) verbunden sind, fällt dieser Suchprozess umso kürzer aus, je geringer der Preis eines Gutes und damit das Risiko für einen Fehlkauf sind. Die meisten Konsumenten verändern deshalb ihr „relevant set“ erst, wenn

8 Die Präferenzordnungen werden in der mikroökonomischen Theorie als gegeben unterstellt. Die Erklärung, wie sie entstehen bzw. sich verändern (lassen), wird anderen sozialwissenschaftlichen Disziplinen wie der Psychologie und der Soziologie überlassen. Die betriebswirtschaftliche Konsumforschung ist dagegen gerade an dieser Frage interessiert (vgl. Kroeber-Riehl et al. 2011). 
sich entweder der Preis oder die wahrgenommene Qualität eines Angebotes - oder aber ihr Einkommen - spürbar verändert haben. ${ }^{9}$

Der grundlegende Analyserahmen des mikroökonomischen Konsummodells bleibt von begrenzt rationalem Verhalten aber letztlich unberührt. Ein solches Verhalten impliziert lediglich, dass immer nur die jeweils bekannten Auswahlalternativen in die Entscheidungen eines Konsumenten über die Allokation seines Geldbudgets - oder aber die eines Rezipienten über die Allokation seines Zeitbudgets - eingehen.

\subsection{Modellübertragung auf Entscheidungen über die Verwendung verfügbarer Zeitmengen}

Dieses theoretische Modell zur Erklärung der Kosumbudgetallokation lässt sich auch auf die Verwendung knapper Zeitbudgets übertragen. Es gibt aus der Sicht eines Rezipienten gleichwertige Zeitdauer-Kombinationen für zwei Mediennutzungsaktivitäten, die sich grafisch für verschiedene Gesamtnutzenniveaus jeweils als konkav geformte sog. Indifferenzkurven darstellen lassen (Abb. 1). Die Krümmung der Kurve zeigt an, in welchem Verhältnis eine Aktivität jeweils durch die andere ersetzt werden kann, ohne dass sich der Gesamtnutzen beider Aktivitäten ändert. Dies gilt auch für eine bestimmte Art der Mediennutzung (z. B. fernsehen) und eine nichtmediale Aktivität (z. B. spazieren gehen oder Sport treiben). Dieses Austauschverhältnis hängt bei jedem Individuum letztlich von den subjektiv empfundenen Nutzenniveaus der beiden Aktivitäten ab sowie vom Tempo, mit dem bei den einzelnen Aktivitäten subjektive Sättigungseffekte auftreten. Die Präferenzordnung jedes Individuums lässt sich insofern als Schar von Indifferenzkurven bzw. partiellen Substitutionsbeziehungen zwischen allen möglichen Aktivitäten abbilden.

Das verfügbare Zeitbudget für zwei Aktivitäten lässt sich als sog. Budgetgerade darstellen. Die Steigung der Gerade bildet die jeweiligen Preisrelationen ab, die Entfernung vom Ursprung die für Aktivitäten verfügbare Menge an Zeiteinheiten (z. B. Minuten), über dessen Verwendung ein Rezipient entscheiden kann. Zeit ist nicht handelbar und hat deshalb auch keinen einheitlichen Marktpreis. Die Zeitkosten von Aktivitäten lassen sich deshalb nur auf Basis theoretischer Überlegungen bestimmen: Man kann entweder unterstellen, dass es in dieser Hinsicht keine Unterschiede zwischen einzelnen Aktivitäten gibt (ihre „Zeitpreise“ sind gleich). Die Zeitbudgetgerade hat dann immer die gleiche negative Steigung von $45 \mathrm{Grad}$. Man kann aber auch argumentieren, dass sich Mediennutzungsaktivitäten beispielsweise im Hinblick auf das notwendige Aufmerksamkeitsniveau für die kognitive Verarbeitung von Inhalten unterscheiden, und deshalb Zeit für eine Mediennutzungsaktivität, die einen hohen Grad an Aufmerksamkeit erfordert, als „teurer“ empfunden wird, als Zeit für eine Mediennutzungsaktivität, die weitere nutzenbringende Paralleltätigkeiten zulässt. Die Zeitbudgetgerade wäre dann - je nach „Zeitpreisrelation“ der beiden betrachteten Aktivitäten - entsprechend flacher oder steiler. ${ }^{10}$

Die optimale Zeitaufteilung eines (beschränkt) rational handelnden Rezipienten erfolgt dann in Abhängigkeit von der ihm insgesamt verfügbaren Zeitmenge immer so,

9 Ein begrenzt rational handelnder Konsument verhält sich dann als „Satisfizierer“ statt als „Nutzenmaximierer“ (vgl. u. a. Simon 1959; Kiefer 2005: 231). Dieses modifizierte Verhaltensmodell des homo oeonomicus ist auch als RREEMM-Modell (resourceful restricted evaluating expecting maximising man) bekannt (Frey 2004: 33).

10 Becker (1965) schlägt einen Opportunitätskostenansatz vor, bei dem die Zeit individuell unterschiedlich ist und von der jeweiligen Lohnhöhe bestimmt wird. 
Abbildung 1: Optimale Zeitaufteilung zwischen zwei Aktivitäten bei gegebenen Zeitkosten und Zeitbudget

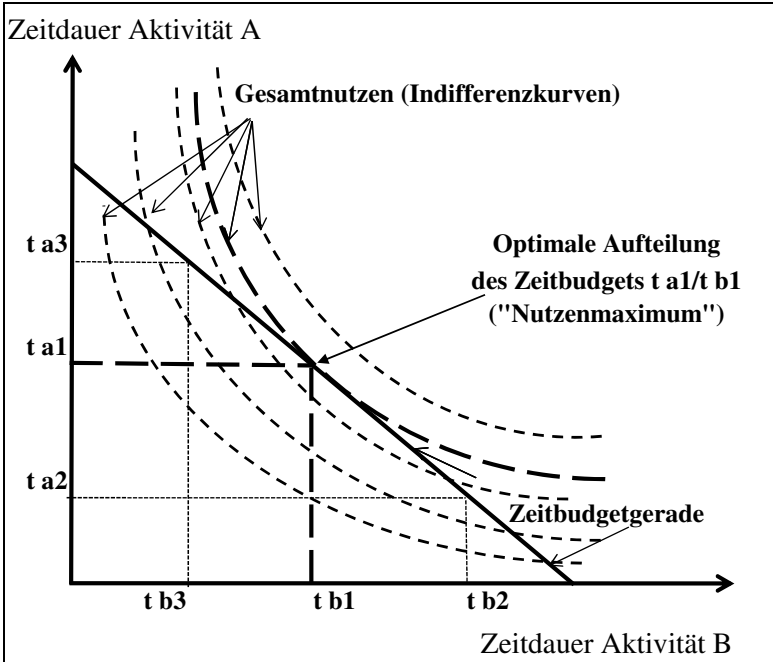

Quelle: Eigene Darstellung

dass er sein höchst mögliches Gesamtnutzenniveau erreicht. Dieses Nutzenoptimum entspricht dem Tangentialpunkt der Zeitbudgetgeraden mit der am weitesten vom Ursprung entfernten Indifferenzkurve (in Abb. 1 die Kombination ta1tb1 anstelle von ta2tb2 oder ta3tb3). Dieses Nutzenoptimum ist gleichzeitig der Zustand, in dem der subjektiv empfundene Nutzen der jeweils letzten Minute aller Aktivitäten gleich ist (bzw. ihren relativen Zeitkosten entspricht).

Unabhängig davon, ob man gleiche oder unterschiedliche Zeitkosten für einzelne Mediennutzungs- und nichtmediale Aktivitäten unterstellt, ändert sich die optimale Aufteilung des Zeitbudgets immer dann, wenn sich die Menge der insgesamt frei disponierbaren Zeiteinheiten verändert. Sofern diese Menge zwischen einzelnen Tagen und Tagesabschnitten variiert, wird sich auch das Verhältnis der Aktivitätsdauern entsprechend verändern. Sofern dabei ein niedrigerer Anteil einer Aktivität systematisch mit einem höheren Anteil einer anderen Aktivität einhergeht, besteht eine Substitutionsbeziehung.

\section{Bedeutung der Begriffe Substitution und Komplementarität in Kommunikationswissenschaft und Ökonomie}

Während damit der Begriff der Substitution in Kommunikationswissenschaft und Ökonomie den gleichen Bedeutungsgehalt haben, ist dies beim Begriff der Komplementarität nicht der Fall (vgl. a. Hagen 1998: 107; Mögerle 2009: 120f.). Ein wesentlicher Unterschied besteht im Verständnis des Zusammenhangs zwischen den subjektiven Bewertungen von Gütern bzw. Aktivitäten und ihren substitutiven und komplementären Beziehungen.

In der Ökonomie werden als Komplementärbeziehungen ausschließlich Fälle verstanden, bei denen erst die gemeinsame Nutzung zweier Konsumgüter dem Konsumen- 
ten einen subjektiven Nutzengewinn erbringt. Übertragen auf die Durchführung zweier Aktivitäten ist eine „komplementäre Nutzung“ zweier Medien nur dann gegeben, wenn eine längere Nutzungsdauer einer Mediennutzungsaktivität systematisch mit einer längeren Nutzungsdauer einer zweiten Mediennutzungsaktivität einhergeht. Komplementarität in einer ökonomischen Bedeutung wäre damit dann gegeben, wenn die The-morethe-more-Regel gilt, die in der Frühphase der Verbreitung des Radios von Lazarsfeld et al. (1948) gegenüber gedruckten Medien aufgestellt wurde.

In der Kommunikationswissenschaft wird häufig bereits ein reines Nebeneinander unterschiedlicher Mediennutzungsaktivitäten als komplementäre Nutzung bezeichnet. Dies gilt auch für aktuelle Konzepte medienübergreifenden Nutzungsverhaltens, beispielsweise diejenigen individueller Medienmenüs (Haas 2007) oder individueller Medienrepertoires (Hasebrink/Popp 2006; Hasebrink/Domeyer 2012). Dieses Verständnis des Begriffs Komplementarität erklärt sich teilweise dadurch, dass Nutzungsepisoden verschiedener Medienangebote - entsprechend der Annahme medienspezifischer Gratifikationen im U\&G-Ansatz - als sich ergänzende komplementäre Tätigkeiten angesehen werden, die Rezipienten im Hinblick auf ihre jeweilige Bedürfnisstruktur optimieren.

Auf den ersten Blick entspricht das U\&G-Konzept der Mediengratifikationen dem Nutzenkonzept der Ökonomie (vgl. Picard 1989). Rezipienten treffen medien- bzw. aktivitätsübergreifende Nutzungsentscheidungen nach den von ihnen subjektiv erwarteten Gratifikationen bzw. dem von ihnen erwarteten Nutzen. Diese Gratifikationsbzw. Nutzenerwartungen sind jeweils Ergebnis bereits gemachter Erfahrungen (erhaltener Gratifikationen) mit Mediengattungen, Genres oder einzelnen Medienprodukten. Allerdings gibt es zwischen den beiden Konzepten zwei wesentliche Unterschiede: Zum einen werden vom U\&G-Ansatz die Aspekte der begrenzt verfügbaren Zeitressourcen und der mit Mediennutzungsaktivitäten verbundenen Kosten als eigenständige Einflussfaktoren auf die Medienwahl nicht berücksichtigt. Zum anderen wird dort die Austauschbarkeit bzw. die Ersetzung eines Medienangebotes durch ein anderes allein daran festgemacht, ob beide auf der gleichen Gratifikationsdimension Nutzen stiften.

Eine Ausnahme im Hinblick auf eine simultane Berücksichtigung von Nutzenund Kostenaspekten bei Medienauswahlentscheidungen ist die Theorie der Nische, die auf einer Übertragung evolutionstheoretischer Konzepte auf die Medienentwicklung basiert (Dimmick 1993, 2003). ${ }^{11}$ Der Grad der Überlappung auf den verschiedenen Gratifikationsdimensionen bestimmt zwar auch hier das Substitutionspotenzial zwischen alter und neuer Mediengattung. Es muss aber nicht zu einer völligen Verdrängung kommen, sofern es dem insgesamt unterlegenen Medium gelingt, sich an den veränderten intermedialen Wettbewerb anzupassen und auf solche Gratifikationsnischen zu spezialisieren, in der es weiterhin überlegen ist. Überlegenheit wird dabei so verstanden, dass für den Rezipienten mit dem gleichen Zeitaufwand eine höhere Leistung oder mit einem niedrigeren Zeitaufwand die gleiche Leistung verbunden ist. Als Beispiel gilt die auf eine stationäre und mobile Nebenbeinutzung ausgerichtete Anpassung von Radioprogrammen nach dem Auftreten des Fernsehens.

11 Das von Meulemann (2012: 244) zur Erklärung von Substitutionsbeziehungen zwischen Mediengattungen vorgeschlagene Konzept psychischer Kosten erweitert das in der Ökonomie und im U\&G-Ansatz verwendete Präferenzkonzept um einen Kostenbegriff. Eine KostenNutzen-Abwägung im ökonomischen Sinn wird damit jedoch nicht beschrieben. Die Rangordnung zwischen verschiedenen Mediennutzungs- und nichtmedialen Aktivitäten ist vielmehr in erster Linie vom damit verbundenen emotionalen und kognitiven Aufwand abhängig und nicht mehr vom jeweils erwarteten (positiven) Nutzen. 
Auch in der ökonomischen Theorie gibt es im Zusammenhang mit Veränderungen der Konsumnachfrage aufgrund von Preisänderungen die Vorstellung, dass Güter mit ähnlichen Funktionalitäten eher untereinander ersetzt werden als solche mit verschiedenen Funktionalitäten. Der Grad einer aus der Sicht der Nachfrager bestehenden Produktähnlichkeit (Bedarfsmarktkonzept) ist ein zentrales Kriterium, um zwei Märkte voneinander abzugrenzen (Seufert/Gundlach 2012: 335). Auf aggregierter Ebene lässt sich dabei als quantitatives Maß für den Grad der Substituierbarkeit die Kreuz-PreisElastizität der Nachfrage verwenden, die das Ausmaß der mengenmäßigen Nachfrageänderung aller Konsumenten für ein Gut bei der Preisänderung eines zweiten Gutes beschreibt. $^{12}$

Wie oben bereits erläutert, unterstellt die Mikroökonomie aber nicht nur einen Preiseffekt, der zu Umschichtungen im Konsumbudget führt, sondern auch Nachfrageverschiebungen als Folge eines Einkommenseffekts. Hierbei spielt das Argument gleicher oder ungleicher Funktionalitäten keine Rolle. Vielmehr werden die in der Regel für verschiedene Konsumgüter unterschiedlichen Nachfragereaktionen mit der Existenz einer Bedürfnishierarchie erklärt. Erst wenn bei den Grundbedürfnissen ein Sättigungseffekt eintritt, werden auch Güter zur Befriedigung „höherer Bedürfnisse“ in größeren Mengen gekauft. Mit steigenden Einkommen sinkt deshalb der Anteil von Gütern zur Grundbedürfnisbefriedigung an der Gesamtmenge aller gekauften Güter, teilweise geht ihre Nachfragemenge sogar absolut zurück (inferiore Güter). Dagegen nimmt der Anteil von hochwertigen Verbrauchs- und Gebrauchsgütern bis hin zu Luxusgütern zusammen mit dem Einkommen zu (superiore Güter). Als quantitatives Maß für die Stärke und Richtung dieses Einflusses lässt sich auf aggregierter Ebene die Einkommenselastizität der Nachfrage verwenden. ${ }^{13}$

Übertragen auf das Modell der Zeitallokation wäre die Zeitelastizität einer Aktivität die vergleichbare Maßgröße. Verschiebungen zwischen den Zeitdauern bzw. Zeitanteilen zweier Mediennutzungsaktivitäten oder zu nichtmedialen Aktivitäten können deshalb auch auftreten, ohne dass ähnliche Informations- oder Unterhaltungsbedürfnisse befriedigt werden müssen.

Abbildung 2 verdeutlicht, dass die Stärke dieser Art von Substitutionsbeziehungen zwischen Aktivitäten zum einen vom jeweiligen Nutzenniveau einer Aktivität und zum

12 Der KPE-Wert beschreibt das Ausmaß der prozentualen Veränderung der aggregierten Nachfragemenge eines Gutes A in Reaktion auf eine Preisänderung um x Prozent bei einem anderen Gut B. Im Fall partiell substitutiver Güter gilt KPE >0, da der durch die Preiserhöhung induzierte Nachfragerückgang bei Gut B aus der Sicht eines Teils der Konsumenten durch Gut A kompensiert werden kann. Je höher der KPE-Wert, desto höher ist der Substitutionsgrad. Beim KPE-Wert von 1 handelt es sich um perfekte Substitute, d. h. alle Konsumenten halten A für einen adäquaten Ersatz von B und die gesamte Nachfrage würde zum preiswerteren Gut A wechseln. Im Fall komplementärer Güter gilt dagegen $\mathrm{KPE}<0$, da der durch die Preissteigerung induzierte Nachfragerückgang bei einem Gut A auch beim komplementären Gut B zu einem Nachfragerückgang führt (vgl. Varian 2007: 105). Da es keine bewährte Methode zur Messung relativer Zeitkosten für einzelne Aktivitäten und damit auch nicht für deren Änderungen gibt, lässt sich die Stärke der Substitutionsbeziehungen zwischen zwei Mediennutzungsaktivitäten oder zu nichtmedialen Aktivitäten auf diese Art nicht sinnvoll schätzen.

13 Die Einkommenselastizität $(E E)$ ist als prozentuale Veränderung der Nachfragemenge nach Gut A bei einer Einkommenserhöhung von x Prozent definiert. Sie kann folgende Wertebereiche annehmen: Falls EE $>1$ handelt es sich um superiore Güter. Falls $0<\mathrm{EE}<1$ handelt es sich um relativ inferiore Güter, d. h. die Nachfragemenge des Gutes nimmt zwar zu, sein Anteil am Konsumbudget geht aber zurück. Falls $\mathrm{EE}<0$ handelt es sich um absolut inferiore Güter, d. h. die Nachfragemenge des Gutes geht zurück (vgl. Varian 2007: 90f.). 
Abbildung 2: Einfluss von Nutzenniveaus und Sättigungseffekten verschiedener Aktivitäten auf die Zeitallokation

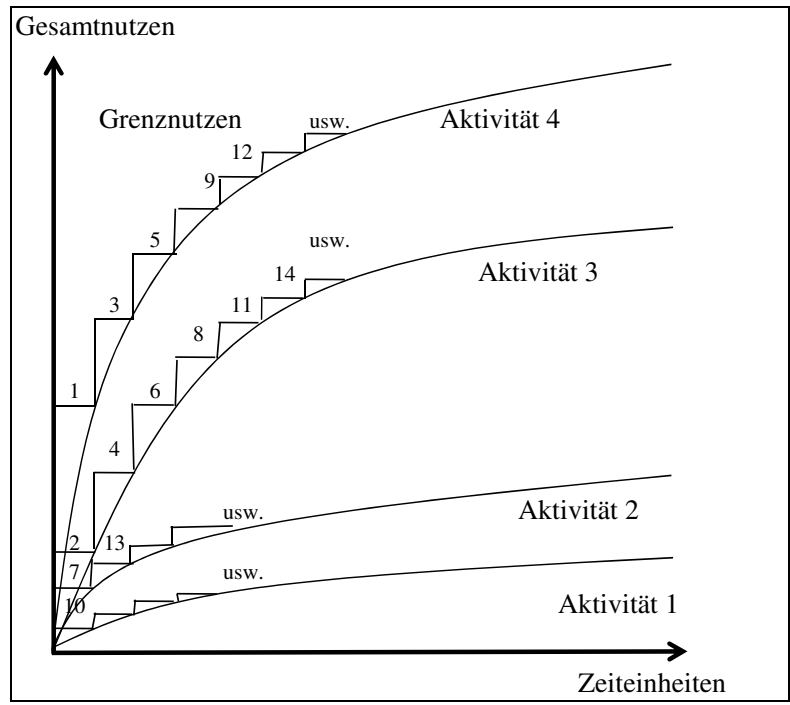

Quelle: Eigene Darstellung

anderen vom Tempo, mit dem bei ihr Sättigungseffekte eintreten, abhängt. Bei einem insgesamt eher geringen Zeitbudget werden sich mehr Zeiteinheiten auf wenige Aktivitäten mit subjektiv hohem Nutzenniveau und geringen Sättigungseffekten konzentrieren. Bei insgesamt höherem Zeitbudget werden die Zeiteinheiten auf insgesamt mehr Aktivitäten aufgeteilt, da nun auch solche Aktivitäten ausgeführt werden, deren (Anfangs-)Nutzenniveau zwar niedriger ist, aber immer noch höher als der Nutzengewinn, der aus der Fortsetzung von Aktivitäten gezogen werden kann, weil sich ihr (Grenz-) Nutzen aufgrund von Sättigungseffekten stark reduziert. So würden in Abbildung 2 bei einer Zeitmenge von bis zu sechs verfügbaren Zeiteinheiten nur die Aktivitäten 4 und 3 ausgeführt, ab der siebten Zeiteinheit dann auch Aktivität 2 und schließlich ab der zehnten Zeiteinheit auch Aktivität 1.

\section{Forschungsfragen und methodisches Vorgehen}

\subsection{Forschungsfragen}

Der vorliegende Ansatz geht davon aus, dass sich die Medienauswahl im Rahmen eines solchen mikroökonomisch fundierten Modells der Zeitallokation medienübergreifend erklären lässt. Danach wird die individuelle Nutzungsdauer einzelner Mediengattungen (sowie die Dauer der für nichtmediale Tätigkeiten aufgewendeten Zeit) einerseits durch längerfristig veränderbare Präferenzordnungen (subjektive Rangordnungen zwischen Aktivitäten nach ihrem jeweils subjektiv empfundenen Nutzenbeitrag) und andererseits durch situative Einflüsse, die sich in kurzfristig variablen Zeitstrukturen (Dauer der individuell verfügbaren Freizeit bzw. von weniger frei disponibler Zeit) zeigen, beeinflusst. Entsprechend sollen folgende Forschungsfragen beantwortet werden: 
Forschungsfrage 1: In welchem Umfang hat die Verbreitung der „neuen“ Mediengattung Internet bislang zu geringeren Nutzungsdauern bzw. niedrigeren Präferenzstärken für „alte“ Mediengattungen/Aktivitäten geführt? (Stärke der langfristigen Substitutionseffekte).

Forschungsfrage 2: In welchem Umfang geht aktuell die höhere Nutzungsdauer einer Mediengattung mit einer geringeren Nutzungsdauer anderer Mediengattungen/Aktivitäten einher? (Stärke der kurzfristigen Substitutionseffekte).

\subsection{Spezifizierung eines Zeitallokationsmodells für unterschiedliche Zeitarten}

Die Grundstruktur eines Strukturgleichungsmodells, das auf Basis eines Zeitallokationsmodells der Medienauswahl entwickelt wird, muss die wesentliche Annahme berücksichtigen, dass sich die relativen Präferenzen einer Person für einzelne Aktivitäten (ihre relative Wertschätzung für verschiedene Mediennutzungs- und nichtmediale Aktivitäten) nicht direkt in den relativen Nutzungsdauern widerspiegeln, da diese immer auch von der jeweils gegebenen Zeitrestriktion abhängen. Das Grundmodell für jede (Mediennutzungs-)Aktivität besteht damit aus der endogenen Variablen Aktivitätsumfang und zwei exogenen latenten Variablen Aktivitätspräferenz und Zeitverfügbarkeit (vgl. Wilhelm/Seufert 2011: 114).

Für die Messmodelle dieser drei Variablen müssen geeignete Indikatorvariablen gefunden werden. Da die Spezifikation und Schätzung der Strukturgleichungsmodelle sekundäranalytisch erfolgte, hat sich die Spezifizierung der Messmodelle an den Informationen zur Zeitverwendung orientiert, die in den zur Schätzung verwendeten Datensätzen enthalten sind. Genutzt wurden primär die repräsentativ erhobenen Daten der Langzeitstudie Massenkommunikation (MK) aus den Jahren 1995, 2005 und 2010, denen jeweils eine ähnliche Erhebungsmethodik zugrunde liegt. In ihnen sind auf Basis von Tagesablaufinterviews erhobene Nutzungsdauern der acht Mediengattungen Fernsehen, Video, Hörfunk, Tonträger, Zeitung, Zeitschrift, Buch und Internet (1995: PC-Nutzung) sowie Informationen zur Dauer allgemeiner Tätigkeiten enthalten. ${ }^{14}$

\section{Unterscheidung von Mediennutzungsaktivitäten in drei Zeitarten}

Die dort erfassten allgemeinen Tätigkeiten ermöglichen eine getrennte Analyse des Mediennutzungsverhaltens in drei verschiedenen Zeitarten. Neben der Zeitverwendung in der Freizeit und Arbeitszeit wurde diese auch für eine dritte Zeitart Reproduktionszeit analysiert. Damit wurden Erkenntnisse der soziologischen und psychologischen Freizeitforschung berücksichtigt, wonach eine Gleichsetzung von Freizeit mit arbeitsfreier Zeit zu kurz greifen würde. Für die Kategorie Freizeit ist ihre hohe Disponibilität charakteristisch. Es gibt jedoch eine Reihe von Tagesabschnitten, die zwar arbeitsfrei (bzw. ausbildungsfrei) sind, aber aufgrund vielfältiger Verpflichtungen subjektiv nicht als Freizeit wahrgenommen werden (u. a. Scheuch 1972: 30). In der Literatur findet man eine Vielzahl von Begrifflichkeiten für diese weder der Arbeitszeit noch der Freizeit zuordenbaren Zeitabschnitte. Opaschowski (2008: 164) und Meulemann (2012: 253) verwenden den Begriff der Obligationszeit, Lüdtke (1984: 346) den der Verpflichtungszei-

14 Zur Kreuzvalidierung wurden auch Daten der Media Analyse verwendet, in denen die Nutzungsdauern der Printmedien im Rahmen des Tagesablaufinterviews aber nicht mit erhoben werden. 
ten. In den Auswertungen der ARD/ZDF-Langzeitstudie Massenkommunikation findet sich eine Abgrenzung zwischen Produktionszeit und Regenerationszeit (Ridder/Turecek 2011: 571).

Die Verwendung des Begriffs Reproduktionszeit soll auch den Unterschied zur kategorialen Abgrenzung dieser anderen Autoren deutlich machen. Ihr werden im Folgenden fünf der dreizehn in Deutschland in Tagesablaufinterviews üblicherweise erhobenen allgemeinen Tätigkeiten (Körperpflege, Mahlzeiten zuhause, Hausarbeit, Einkäufe, Wegezeiten) zugeordnet, die für die Lebensführung zwingend notwendig sind. Die Zeitart Freizeit ist dann eine Restkategorie mit allen nicht der Arbeitszeit oder der Reproduktionszeit zugeordneten Tätigkeiten (Wilhelm/Seufert 2011: 113). ${ }^{15}$ Die Zusammenfassung dieser fünf Tätigkeiten zu einer eigenen Zeitart Reproduktionszeit lässt sich auch damit begründen, dass dabei jeweils nur eine Nebenbeinutzung mit niedrigerer Aufmerksamkeit möglich ist, während die Nutzung in der Freizeit mit voller Aufmerksamkeit erfolgen kann (wenn auch nicht muss). Die Rangfolge der Präferenzen für einzelne Mediengattungen ist deshalb in der Reproduktionszeit mit hoher Wahrscheinlichkeit eine andere als in der Freizeit.

\section{Spezifizierung der Messmodelle}

Für die endogene Variable Aktivitätsumfang ist die jeweilige Dauer der Aktivität in Minuten das geeignetste Messmodell. Die Tagesablaufdaten der ARD/ZDF-Langzeitstudie Massenkommunikation enthalten für jede Viertelstunde von 5 bis 0 Uhr Informationen darüber, im Rahmen welcher allgemeinen Aktivitäten der Befragte bestimmte Medien genutzt hat. Entsprechend konnten die Nutzungsdauern aller Mediengattungen in Minuten in den drei definierten Zeitarten (Freizeit, Reproduktionszeit, Arbeitszeit) berechnet werden.

Für das Messmodell der exogenen latenten Variable Zeitverfügbarkeit wurden die verfügbaren Zeitmengen einer Zeitart in verschiedenen Tagesabschnitten als Indikatorvariablen verwendet. Hierfür erfolgte eine Unterteilung in folgende sechs Abschnitte (vgl. dazu auch Fritz/Klingler 2003: 12): 5 bis 8 Uhr, 8 bis 12 Uhr, 12 bis 14 Uhr, 14 bis $17 \mathrm{Uhr}, 17$ bis $20 \mathrm{Uhr}$ und 20 bis 0 Uhr. Durch eine solche Differenzierung lässt sich neben den insgesamt verfügbaren Mengen der einzelnen Zeitarten auch die Variabilität ihrer zeitlichen Verteilung im Tagesablauf abbilden. Dabei wurden die Konstrukte „verfügbare Freizeit" (gemessen über die vier Zeitabschnitte 8 bis 12 Uhr, 12 bis 14 Uhr, 14 bis 17 Uhr und 17 bis $20 \mathrm{Uhr}$ ) und „verfügbare Reproduktionszeit“ (gemessen über die drei Zeitabschnitte 8 bis $12 \mathrm{Uhr}, 12$ bis $14 \mathrm{Uhr}$ und 14 bis $17 \mathrm{Uhr}$ ) als jeweils reflektive Faktoren behandelt (Wilhelm/Seufert 2011: 118). ${ }^{16}$

15 Die auf Tagebuchaufzeichnungen gestützte Zeitbudgeterhebung des Statistischen Bundesamtes von 2001/2002 erfasst Haupt- und Nebenaktivitäten sehr viel differenzierter. Die Hauptaktivität wird nach folgenden neun Oberkategorien geordnet: physiologische Regeneration, Erwerbstätigkeit, Qualifikation/Bildung, Haushaltsführung/Familienbetreuung, ehrenamtliche Tätigkeiten, soziales Leben/Unterhaltung, Hobbys/Spiele, Massenmedien und Wegezeiten (Ehling et al. 2001: 435).

16 Folgende Kriterien müssen bei einer reflektiven Faktorenbildung berücksichtigt werden (Homburg et al. 2008a: 294): (1) Die Konstrukte sind kausale Ursachen ihrer Indikatoren: Eine Zunahme der Freizeit (z. B. am Wochenende) führt in der Regel zu einer Zunahme der Freizeit in allen hier berücksichtigten Zeitabschnitten. (2) Die Indikatoren haben ein inhaltlich ähnliches Thema (verfügbare Freizeit- bzw. Reproduktionszeitmenge). (3) Es können Korrelationen zwischen den Indikatoren erwartet werden. (4) Im überwiegenden Fall kann angenommen 
Das Messmodell für die latente Variable Aktivitätspräferenz wurde über relative Zeitanteile spezifiziert: Zur Aufdeckung der relativen Stärke der Präferenzen für einzelne Mediengattungen wurden die Nutzungsdauer der acht erfassten Mediengattungen (TV, Radio, Tonträger, Internet, Video/DVD, Zeitung, Zeitschrift und Buch) zueinander ins Verhältnis gesetzt. Dies ergibt insgesamt 56 Nutzungsrelationen, die in eine Faktorenanalyse eingingen, welche entsprechend der acht Medien eine 8-Faktoren-Lösung ergab. Diese acht Faktorwerte können als Präferenzstärken interpretiert werden, die die Rangfolge der Wertschätzung für einzelne Mediennutzungsaktivitäten zum Ausdruck bringen. ${ }^{17}$ So wurde beispielsweise die Präferenz für Fernsehen in der Freizeit über acht Indikatoren, die jeweils das Verhältnis der Fernsehnutzungsdauer zur Nutzungsdauer der anderen erfassten Medien (Radio, Tonträger, Internet, Video/DVD, Zeitung, Zeitschrift und Buch) abbilden, gemessen. Analog wurden außerdem Faktoren zu Präferenzen für nichtmediale Aktivitäten ermittelt. Daraus ergaben sich die beiden Faktoren „Präferenz für nichtmediale Aktivitäten im Haus“ (sonstige Freizeit im Haus) und „Präferenz für nichtmediale Aktivitäten außer Haus" (Freunde treffen, Kneipen- und Restaurantbesuche, sonstige Freizeit außer Haus). ${ }^{18}$

\section{Modellschätzungen}

Zur Schätzung der relativen Einflussstärken von Zeitrestriktionen (latente exogene Variable „Zeitverfügbarkeit“) und Präferenzstärken (latente exogene Variable „Medienpräferenz") auf die Dauer einzelner Mediennutzungsaktivitäten (latente exogene Variable „Mediennutzung“") wurden jeweils die entsprechenden Grundmodelle für eine Zeitart herangezogen. Abb. 3 zeigt dies beispielhaft für die Fernsehnutzung in der Freizeit. Die Modellschätzungen für die tagesaktuellen Medien Fernsehen, Hörfunk, Zeitung und Internet sowie für nichtmediale Aktivitäten ergaben dabei überwiegend gute bis sehr gute Passungen. ${ }^{19}$ Die Modelle erklären jeweils etwa 80 Prozent der Varianz in den Nutzungsdauern der Befragten.

werden, dass alle Indikatoren ähnliche Ursachen und Wirkungen haben. Diese Kriterien konnten bei der Freizeit für die Zeitabschnitte von 8 bis $20 \mathrm{Uhr}$ (Kriterien 1 und 3 jedoch nicht für die Abschnitte 5 bis 8 Uhr und 20 bis 0 Uhr) und bei der Reproduktionszeit für die Zeitabschnitte 8 bis $17 \mathrm{Uhr}$ (Kriterien 1 und 3 ebenfalls nicht für die Abschnitte 5 bis $8 \mathrm{Uhr}$ und 17 bis 0 Uhr) als erfüllt gelten. Die Diskriminanzvalidität der Konstrukte konnte nachgewiesen werden. Die Reliabilitätsprüfung auf Indikatoren- und Faktorenebene erfolgte nach Gütekriterien der zweiten Generation in Anlehnung an Homburg et al. (2008b: 568).

17 Hauptkomponentenanalyse mit Varimax-Rotation: Die durch diese acht Faktoren erklärte Gesamtvarianz lag bei 83,1 Prozent. Das Maß der Stichprobeneignung nach Kaiser-MeyerOlkin betrug .84. Auch für die Nutzungsrelationen in der Reproduktionszeit wurde eine Faktorenanalyse berechnet. Die hohen Faktorladungen (>.60) bestätigen Vermutungen über eine klare Abgrenzbarkeit dieser Medienpräferenzen und berücksichtigen dennoch die relative Bedeutung der einzelnen Medien im Sinne eines medienübergreifenden Nutzungsverhaltens. Die acht extrahierbaren Faktoren der Medienpräferenzen konnten jeweils mittels konfirmatorischer Faktorenanalysen bestätigt werden.

18 Die Hauptkomponentenanalyse mit Varimax-Rotation ergab eine Zwei-Faktor-Lösung mit einer erklärten Gesamtvarianz von 82,1 Prozent (KMO = .95; Faktorladungen > .60). Die Faktorstruktur konnte ebenfalls im Rahmen einer konfirmatorischen Faktorenanalyse bestätigt werden.

19 Für die Modelle der nicht-tagesaktuellen Medien Video, Tonträger, Zeitschrift und Buch waren die Passungen nicht ausreichend, da in den verwendeten MK-Datensätzen jeweils nur eine geringe Zahl an Fällen enthalten war. 
Abbildung 3: Modell zur Schätzung des Einflusses von Zeitrestriktionen auf die Nutzung einer Mediengattung (Beispiel: TV-Nutzung in der Freizeit)

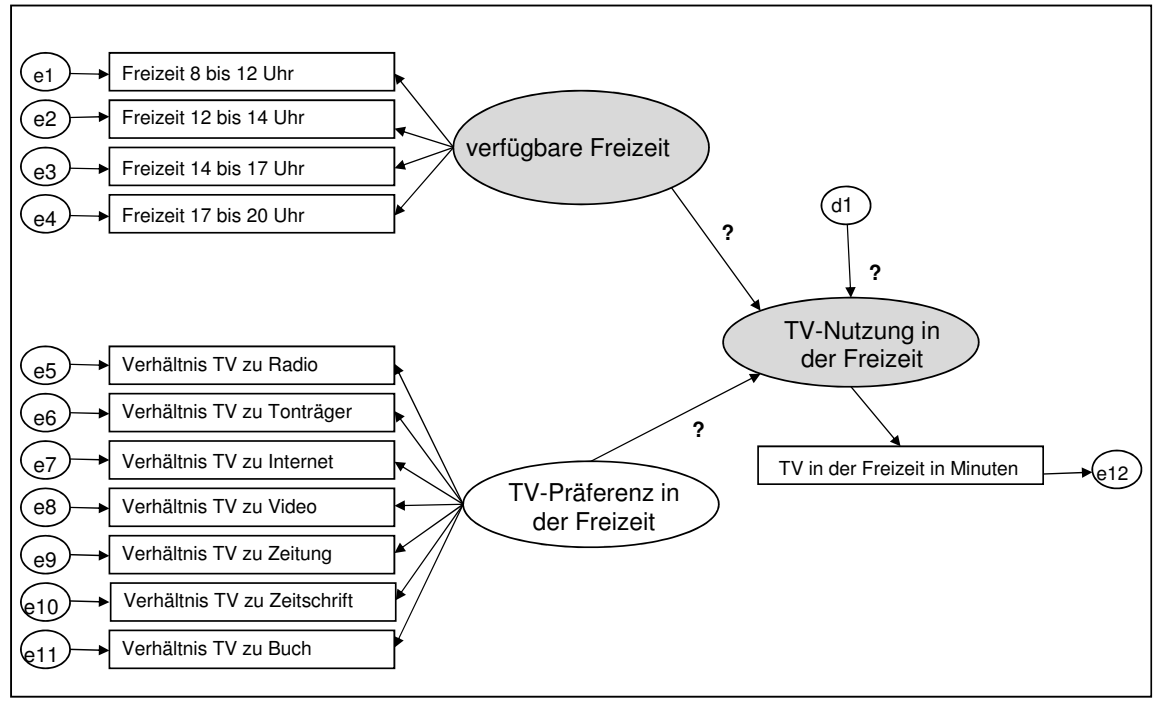

Quelle: Eigene Darstellung

Die Nutzungsdauer aller tagesaktuellen Medien wurde im Erhebungsjahr 2010 jeweils zu wenigstens 20 Prozent von Zeitrestriktionen beeinflusst. Beim Fernsehen in der Freizeit und der Radionutzung in der Reproduktionszeit erklärt die Menge der verfügbaren Zeit sogar jeweils über 50 Prozent der Nutzungsdauer. In der Freizeit hat das Fernsehen entsprechend die stärkste Zeitelastizität, d. h. mit jeder zusätzlichen Minute Freizeit verlängert sich seine Nutzungsdauer um knapp 34 Sekunden und damit weit stärker als die der Mediengattungen Internet, Radio oder Zeitung. In der Reproduktionszeit gilt dies für die Dauer der Radionutzung, die sich mit jeder zusätzlichen Minute Reproduktionsaktivität um über 30 Sekunden verlängert (Wilhelm/Seufert 2011: 121f.).

\subsection{Schätzung der Stärke kurzfristiger Substitutionsbeziehungen auf Basis von Teilmodellen}

Ein Totalmodell, das sämtliche Wechselwirkungen der verschiedenen Zeitrestriktionen und Medienpräferenzstärken auf die Nutzungsdauern der acht erfassten Mediengattungen und die Dauer nichtmedialer Aktivitäten sowie Wechselbeziehungen zwischen Präferenzen abbilden will, enthält für jede der drei Zeitarten elf exogene und neun endogene latente Variablen, so dass die Zahl der zu schätzenden Parameter sehr groß wird. Die exogenen latenten Variablen, die die Aktivitätspräferenzen abbilden, werden über die Relationen der Nutzungsdauern zu allen anderen Mediengattungen bzw. nichtmedialen Aktivitäten gemessen. Deshalb ist außerdem davon auszugehen, dass die Messfehler ihrer Indikatoren teilweise untereinander hoch korrelieren (z. B. der Indikator „Verhältnis Nutzungsdauer TV zu Radio“ für das Konstrukt TV-Präferenz und „Verhältnis Nutzungsdauer Radio zu TV“ für das Konstrukt Radio-Präferenz), da es sich um die jeweils umgekehrten Proportionen handelt. 
Bei der Schätzung kurzfristiger Substitutionsbeziehungen zu einzelnen Erhebungszeitpunkten wurde deshalb nicht mit einem Totalmodell sondern mit Teilmodellen gearbeitet, in denen jeweils das Medienauswahlverhalten für zwei Mediengattungen bzw. für eine Mediengattung und nichtmediale Freizeitaktivitäten spezifiziert und geschätzt wurde. Abbildung 4 zeigt dies beispielhaft für die TV- und Internetnutzung in der Freizeit. Das oben angesprochene Problem der Messfehlerkorrelation kann bei einem Modell mit zwei Konstrukten zu Aktivitätspräferenzen durch Eliminierung der entsprechenden Indikatoren behoben werden. Bei den Konstrukten „TV-Präferenz in der Freizeit" und „Internet-Präferenz in der Freizeit“ im Modell in Abbildung 4 wären dies die Indikatoren „Verhältnis Nutzungsdauer TV zu Internet“ und „Verhältnis Nutzungsdauer Internet zu TV“. Die übrigen Indikatoren (Nutzungsrelationen zu den übrigen Medien) können beibehalten werden.

\section{Abbildung 4: Modell zur Schätzung kurzfristiger Substitutionsbeziebungen zwischen zwei Mediengattungen}

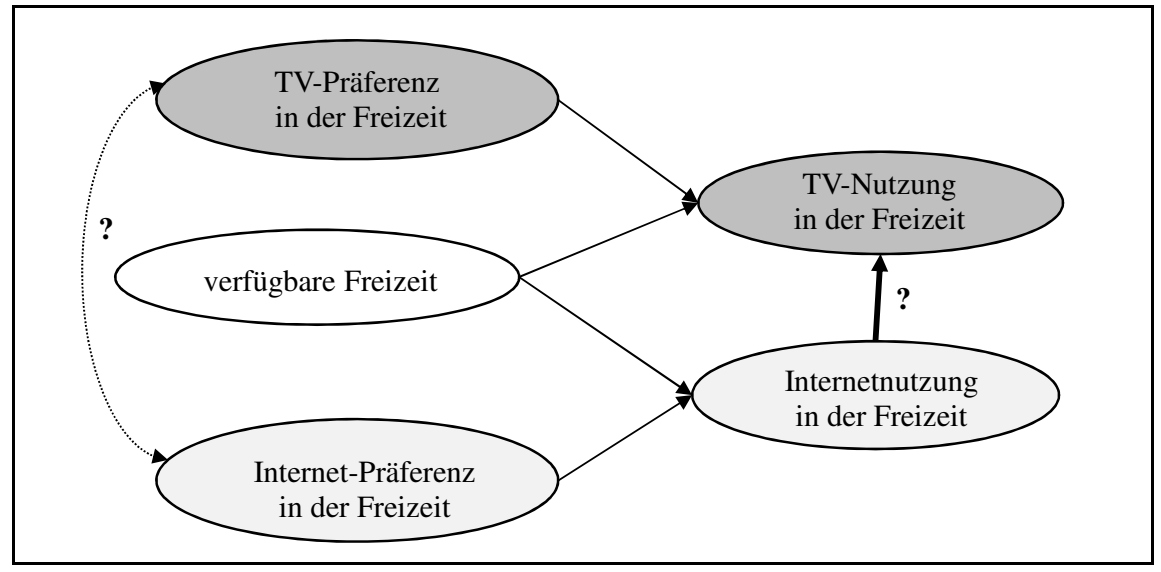

Quelle: Eigene Darstellung

Negative Vorzeichen der geschätzten Regressionsgewichte zwischen den Nutzungsdauern zeigen dann substitutive, positive Regressionsgewichte komplementäre Nutzungsbeziehungen an. Je größer die Regressionsgewichte sind, desto stärker sind diese kurzfristigen Substitutionsbeziehungen. Ein Wert nahe minus 1 würde bedeuten, dass die Nutzungsdauer einer Mediengattung fast ausschließlich von der Nutzungsdauer der anderen Mediengattung abhängig ist, so dass sich bei einer kurzfristigen Änderung der Zeitrestriktionen beide Aktivitäten also jeweils vollständig wechselseitig ersetzen können. Werte nahe Null deuten dagegen auf eine voneinander weitgehend unabhängige Nutzung von zwei Mediengattungen hin.

\subsection{Schätzung der Stärke langfristiger Substitutionseffekte durch Mebrgruppenkausalanalyse}

Empirisch wird häufig aus einem Wachstum des gesamten Zeitbudgets für die Mediennutzung auf ein Nichtvorhandensein von Substitutionseffekten neuer Medien auf alte 
Medien geschlossen (u. a. Oehmichen/Schröter 2007). ${ }^{20}$ Allein aus der Zu- oder Abnahme der durchschnittlichen Nutzungsdauern für verschiedene Mediengattungen im Zeitvergleich lässt sich aber weder auf mögliche langfristige Substitutionseffekte noch auf eventuelle Komplementärbeziehungen zwischen alten und neuen Medien schließen. Eine im Zeitvergleich längere oder kürzere Nutzungsdauer einer Mediengattung muss nicht von Präferenzänderungen, d. h. einer veränderten relativen Wertschätzung für alte Medien nach dem Auftreten eines neuen Mediums verursacht sein, sondern kann auch lediglich die Folge von größeren oder kleineren verfügbaren Zeitmengen sein, beispielsweise einer wachsenden Freizeitmenge (vgl. u. a. Kiefer 1989).

Innerhalb eines mikroökonomisch fundierten Ansatzes können als empirischer Beleg für langfristige Substitutionseffekte eines neuen Mediums geringere Präferenzstärken für alte Medien nach dem Beginn seiner Verbreitung angesehen werden. Im Fall des Internets betrifft dies den Zeitraum von Mitte der 1990er Jahre bis zur Gegenwart. Zur Bestimmung der Stärke solcher Präferenzänderungen wurden auf Basis der oben beschriebenen Grundmodelle für einzelne Mediengattungen Mehrgruppen-Kausalanalysen durchgeführt. Zunächst wurden die mit der gleichen Erhebungsmethodik gewonnenen Datensätze der MK 1995 und 2005 bzw. 2010 als Vergleichsgruppen herangezogen. ${ }^{21}$ Die relativen Unterschiede der Mittelwerte der Präferenzen zwischen der 2005er-Gruppe bzw. der 2010er-Gruppe und der 1995er-Gruppe stehen dann im Fall negativer Abweichungen für die Stärke der langfristigen Substitutionseffekte. Durch die Einbeziehung von drei Erhebungszeitpunkten kann auch überprüft werden, ob diese Veränderungen über den gesamten Zeitraum kontinuierlich stattgefunden haben oder ob in den letzten Jahren eine Verstärkung oder Abschwächung festzustellen ist.

\section{Empirische Ergebnisse}

In der Arbeitszeit werden nur Hörfunk und Internet in nennenswertem Umfang genutzt. Die hier vorgestellten Analysen konzentrieren sich deshalb auf die Stärke von Substitutionsbeziehungen zwischen Mediengattungen in der Freizeit und der Reproduktionszeit. Alle Ergebnisse beziehen sich jeweils auf die Gesamtbevölkerung. ${ }^{22}$

\subsection{Langfristige Substitutionseffekte durch das Internet nach 1995}

Insgesamt hat die durchschnittliche Freizeit pro Tag für Personen ab 14 Jahren in der Zeit von 5 bis 24 Uhr seit Anfang der 1990er Jahre leicht abgenommen: von 495 Minuten im Jahr 1995 auf 455 Minuten im Jahr 2005 bzw. 469 Minuten im Jahr 2010 (Reitze/ Ridder 2011: 80). Allerdings führt die Zunahme von Beschäftigtenverhältnissen mit flexibilisierten Arbeitszeiten zu Umverteilungen der frei disponiblen Zeit über den Tag.

20 Während 1995 noch weniger als 5 Prozent der Bevölkerung das Internet zumindest gelegentlich genutzt haben, waren es im Jahr 2010 bereits 70 Prozent. Die durchschnittliche Internetnutzungsdauer hat am Tag im Jahr 2010 bei 83 Minuten gelegen. Dabei hat das gesamte Zeitbudget für die Mediennutzung von 1995 bis 2010 um 62 Minuten zugenommen.

21 In einer Mehrgruppen-Faktorenanalyse konnte skalare Invarianz nachgewiesen werden. Das bedeutet, dass sowohl die Beziehungsstrukturen als auch die Mittelwerte der Konstrukte für 1995 und 2005 miteinander vergleichbar sind (vgl. Weiber/Mühlhaus 2010: 238).

22 Es wurden auch Mehrgruppenkausalanalysen zur Ermittlung von Unterschieden zwischen sozialen Gruppen durchgeführt, die sich in Alter, formaler Bildung und Erwerbsbeteiligung unterscheiden. Auf diese Ergebnisse wird in einem separaten Beitrag detailliert eingegangen (Seufert/Wilhelm 2013). 
Die Ergebnisse der Mehrgruppen-Kausalanalysen für die Jahre 1995 und 2005 bzw. 2010 zeigen deshalb für die Mittelwerte der exogenen Variable verfügbare Freizeit, die wie oben erläutert über die Freizeitmengen in den Zeitabschnitten von 8 bis 20 Uhr gemessen wird, eine signifikante, wenn auch geringe Erhöhung an.

Die für die Identifizierung langfristiger Substitutionseffekte relevanten Mittelwerte der Präferenzen für die aktuellen Medien Fernsehen, Radio und Zeitung haben sich gegenüber 1995 ebenfalls signifikant und in allen drei Fällen negativ verändert. ${ }^{23}$ Den vergleichsweise stärksten Rückgang der relativen Wertschätzung gegenüber anderen Aktivitäten hat es bei der Zeitungsnutzung gegeben, gefolgt vom Fernsehen und in sehr geringem Umfang beim Radio. Noch stärker als bei diesen drei aktuellen Medien ist der Präferenzmittelwert für zuhause ausgeführte nichtmediale Freizeitaktivitäten zurückgegangen, bei gleichzeitigen höheren Präferenzen für nichtmediale Freizeitaktivitäten außer Haus (Tab. 1). Von 2005 bis 2010 sind dabei kaum noch nennenswerte zusätzliche Veränderungen festzustellen, d. h. die wesentlichen Auswirkungen des Internets auf die Nutzung anderer Mediengattungen waren bereits in den ersten 10 Jahren nach seiner Einführung spürbar.

Tabelle 1: Entwicklung der Präferenzen für aktuelle Medien seit 1995

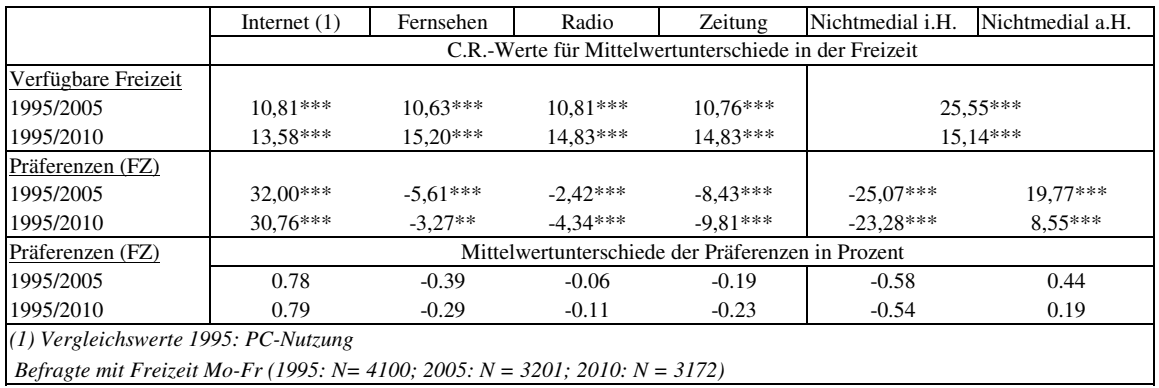

\begin{tabular}{|c|c|c|c|c|c|c|}
\hline & Internet & Fernsehen & Radio & Zeitung & Nichtmedial i.H. & Nichtmedial a.H. \\
\hline & \multicolumn{6}{|c|}{ C.R.-Werte für Mittelwertunterschiede in der Reproduktionszeit } \\
\hline \multicolumn{7}{|l|}{ Reproduktionszeit } \\
\hline $1995 / 2005$ & & $-4,12 * * *$ & $-3,96 * * *$ & $-3,99 * * *$ & & \\
\hline $1995 / 2010$ & & n.s. & n.s. & n.s. & & \\
\hline \multicolumn{7}{|l|}{ Präferenzen (RZ) } \\
\hline $1995 / 2005$ & & $6,11 * * *$ & $23,62^{* * * *}$ & $-3,29 * *$ & & \\
\hline $1995 / 2010$ & & $9,24 * * *$ & $22,09 * * *$ & $-5,06^{* * * *}$ & & \\
\hline Präferenzen (RZ) & \multicolumn{6}{|c|}{ Mittelwertunterschiede der Präferenzen in Prozent } \\
\hline $1995 / 2005$ & & 0.14 & 0.57 & -0.08 & & \\
\hline $1995 / 2010$ & & 0.22 & 0.53 & -0.12 & & \\
\hline
\end{tabular}

Quelle: Eigene Berechnung auf Basis der ARD/ZDF-Langzeitstudie Massenkommunikation Signifikanzniveau: *p<0.05, ** $<0.01, * * \mathrm{p}<0.001$

Der Mittelwert der latenten Variable verfügbare Reproduktionszeit an Wochentagen hat sich von 1995 bis 2005 bzw. 2010 ebenfalls signifikant, aber insgesamt nur marginal verringert. Im gleichen Zeitraum ist vor allem der Präferenzmittelwert für die Radionutzung als Nebenaktivität stark gestiegen. Dies gilt in geringerem Umfang auch für die Fern-

23 Angaben zu globalen Fitmaßen der Modelle finden sich im Anhang (Tab. 3). 
sehnutzung als Nebenaktivität. Während Radio (zuhause und unterwegs) und Fernsehen (zuhause) nebenbei zu allen der Reproduktionszeit zugeordneten Aktivitäten nutzbar sind, ist dies beim überwiegend aktiv zu nutzenden Internetangebot ebenso wie bei der Zeitungslektüre fast nur in den Wegezeiten (solange nicht selbst gefahren wird) oder beim Essen möglich. Die Präferenz für die Zeitungsnutzung als Nebenaktivität hat seit 1995 leicht abgenommen. Inwieweit die Präferenz für die Internetnutzung als Nebenaktivität gleichzeitig zugenommen hat, lässt sich für diesen Zeitraum nicht überprüfen, da die Internetnutzung 1995 aufgrund der damals geringen Nutzerzahlen noch nicht getrennt erfasst wurde.

Insgesamt sind damit die langfristigen Substitutionseffekte des Internets für die übrige Mediennutzung in der Freizeit und der Reproduktionszeit nach 1995 außer bei der Zeitung eher als sehr gering einzustufen. Die zusätzliche Internetnutzung in der Freizeit ist stattdessen stärker „zulasten“ nichtmedialer Aktivitäten gegangen. In der Reproduktionszeit hat sich das Internet bislang kaum negativ auf die Präferenz für andere aktuelle Medien ausgewirkt. Ein Grund ist, dass es aufgrund überwiegend stationärer Nutzung bislang nur in einen Teil der Reproduktionszeit als Nebenaktivität infrage gekommen ist. Dieses Zeitfenster für eine Nebenbeinutzung dürfte sich allerdings in der Zukunft deutlich erweitern, wenn die mobile Internetnutzung von breitbandigen Internetangeboten (Ridder/Engel 2010: 530f.) einen größeren Stellenwert bekommt.

\subsection{Kurzfristige Substitutionsbeziehungen in den Jabren 2005 und 2010}

Wie oben dargestellt, geht der mikroökonomische Ansatz davon aus, dass kurzfristige Substitutionsbeziehungen zwischen Mediennutzungsaktivitäten und zu nichtmedialen Aktivitäten immer latent vorhanden sind. Sie werden sichtbar, wenn situativ infolge von Veränderungen des Handlungsrahmens (größere Zeitknappheit, keine Zugangsmöglichkeit etc.) die eine Mediennutzungsaktivität kurzzeitig durch eine andere ersetzt wird. Tabelle 2 enthält für die Erhebungsjahre 2005 und 2010 die unstandardisierten Regressionsgewichte und die Pfadkoeffizienten (standardisierten Regressionsgewichte) zwischen den Nutzungsdauern der aktuellen Medien in der Freizeit bzw. zur Dauer nichtmedialer Freizeitaktivitäten sowie die Werte der Korrelationen zwischen den Präferenzstärken für die verschiedenen Mediengattungen bzw. für nichtmediale Freizeitaktivitäten im Haus und außer Haus. ${ }^{24}$ Negative Vorzeichen sind jeweils ein Indiz für das Vorhandensein kurzfristiger Substitutionsbeziehungen. Komplementäre Beziehungen müssten sich dagegen in positiven Vorzeichen der unstandardisierten und standardisierten Regressionskoeffizienten oder in positiven Korrelationen zwischen den Präferenzen zeigen.

Aus den Werten der unstandardisierten Regressionskoeffizienten kann direkt abgelesen werden, um wie viele Sekunden sich die Dauer einer Aktivität im Durchschnitt erhöht oder verringert, wenn sich die Dauer der jeweils anderen Aktivität um eine Minute verändert. Die Höhe des Wertes der Pfadkoeffizienten zeigt die relative Stärke dieses Wirkungsstrangs auf die Nutzungsdauer im Vergleich zu den anderen Einflussgrößen. Die Korrelationen zwischen den Präferenzen jeweils zweier Aktivitäten geben an, wie eng im Durchschnitt der Zusammenhang zwischen einer hohen (niedrigen) Präferenz für die Nutzung einer Mediengattung und einer hohen (niedrigen) Präferenz für die Nutzung einer anderen Mediengattung ist.

24 Angaben zu globalen Fitmaßen der Modelle befinden sich im Anhang (Tab. 4 und Tab. 5). 


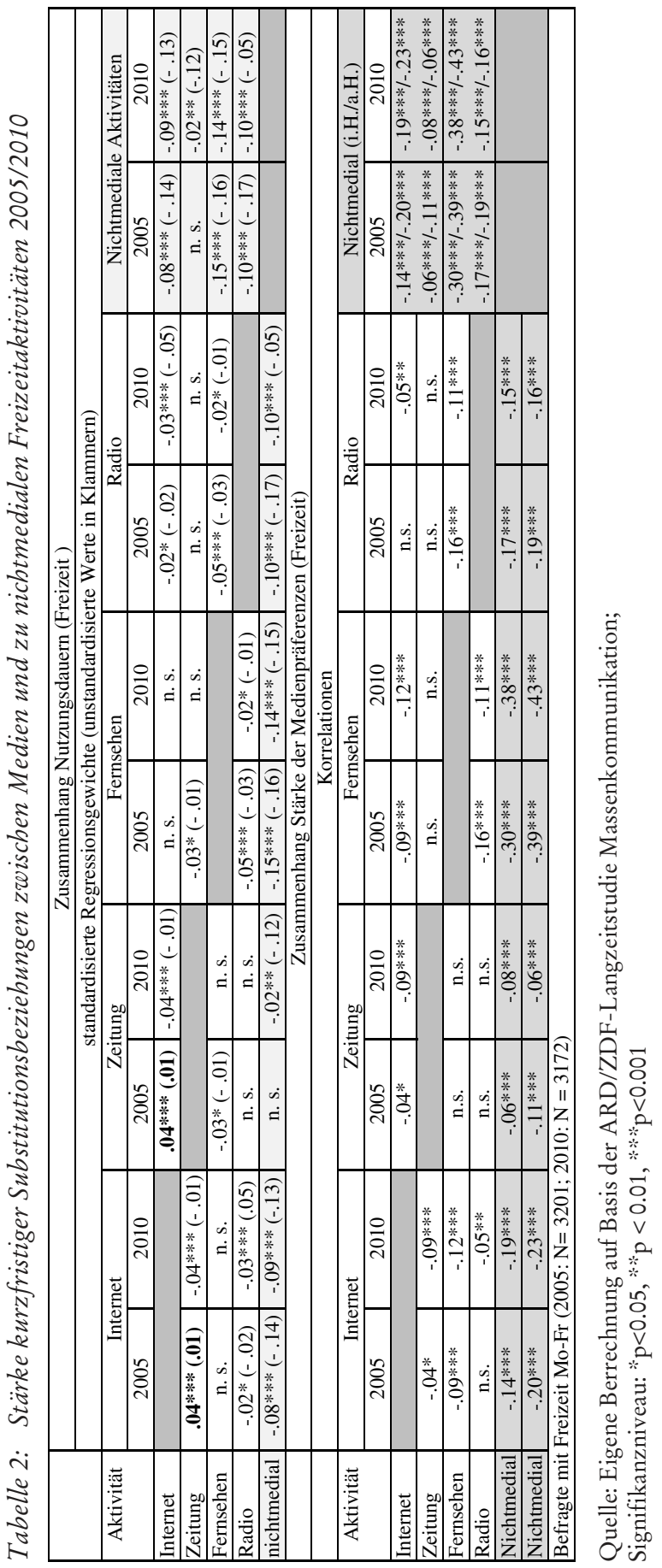


Fast alle statistisch signifikanten Zusammenhänge sowohl für die Pfadkoeffizienten wie für die Korrelationen zwischen den Präferenzen sind negativ. Es findet sich ein einziger Beleg für eine schwache The-more-the-more-Beziehung zwischen Mediengattungen bei den Nutzungsdauern von Zeitungen und Internet im Jahr 2005. Im Jahr 2010 gibt es aber auch hier einen leicht negativen Zusammenhang. Für alle anderen Beziehungen sind zwischen den beiden Jahren kaum nennenswerte Unterschiede auszumachen.

Die negativen Pfadkoeffizienten zwischen den Nutzungsdauern von Internet und Zeitung, Internet und Radio sowie Fernsehen und Radio sind aber durchweg sehr gering. Stärker ausgeprägt sind hingegen bei allen aktuellen Medien die negativen Zusammenhänge zwischen ihren Nutzungsdauern und der Dauer nichtmedialer Freizeitaktivitäten. Am stärksten ist dieser Zusammenhang bei der Fernsehnutzung, am geringsten bei der Zeitungsnutzung.

Wie schwach die kurzfristigen Substitutionsbeziehungen zwischen den Nutzungsdauern aktueller Medien jeweils sind, zeigt sich bei der Umrechnung der unstandardisierten Regressionskoeffizienten in Sekundenwerte (ein Koeffizient von .10 entspricht beispielsweise 6 Sekunden). Im Jahr 2010 hat danach eine Erhöhung der Nutzungsdauer des Internets in der Freizeit um eine Minute im Durchschnitt zu einer Reduzierung nichtmedialer Freizeitaktivitäten um knapp 8 Sekunden, der Radionutzung um 3 Sekunden und der Zeitungsnutzung um weniger als 1 Sekunde geführt. Der 2005 und 2010 jeweils ebenfalls vorhandene leicht negative Zusammenhang zwischen den Nutzungsdauern von Internet und Fernsehen ist statistisch nicht signifikant. Für die Korrelationen zwischen den beiden Präferenzwerten zeigt sich aber ein signifikanter Zusammenhang zwischen jeweils hohen (bzw. niedrigen) Präferenzen für das Internet und niedrigen (bzw. hohen) Präferenzen für das Fernsehen. Die Stärke dieser negativen Korrelation zwischen den Präferenzen für Internet und Fernsehen entspricht damit in etwa der zwischen Internet und Zeitung.

\section{Diskussion}

Die Schätzergebnisse der Strukturgleichungsmodelle zum Einfluss von Zeitverfügbarkeiten und Präferenzen auf die Dauer von Mediennutzungs- und nichtmedialen Aktivitäten deuten sowohl auf langfristige Substitutionseffekte des Internets, die sich in niedrigeren Präferenzen für die alten Medien zeigen, als auch auf kurzfristige Substitutionsbeziehungen zwischen allen Mediengattungen, die in reziproken Nutzungsdauern sichtbar werden, hin. In allen Fällen sind diese aber relativ schwach ausgeprägt und durchweg niedriger als zu nichtmedialen Freizeitaktivitäten im und außer Haus. Eine Komplementarität zwischen Mediengattungen im ökonomischen Sinn, d. h. gemeinsam steigende bzw. sinkende Nutzungsdauern oder Nutzungsanteile zweier Mediengattungen, gibt es generell nicht. Lediglich für die Nutzung von Internet und Zeitung im Jahr 2005 lässt sich eine schwache The-more-the-more-Beziehung feststellen, die im Jahr 2010 nicht mehr bestand.

Diese geringen Effekte sind teilweise auch Folge der Art und Weise, wie in der Kommunikationswissenschaft Mediengattungen voneinander abgegrenzt werden. Von den heute in der ARD/ZDF-Langzeitstudie Massenkommunikation unterschiedenen Mediengattungen (Hörfunk, Fernsehen, Tonträger, Video, Kino, Zeitung, Zeitschrift, Buch und Internet) wurden drei nach dem 2. Weltkrieg jeweils als „neue Medien“ betrachtet und intensiv im Hinblick auf mögliche substitutive Wirkungen diskutiert: Fernsehen ab Mitte der 1960er Jahre, Video ab Anfang der 1980er Jahre und das Internet ab Mitte der 1990er Jahre. Für den Status eines „neuen Mediums“ war dabei eine technische Innova- 
tion offensichtlich allein nicht ausreichend. Die Ablösung des Analog-TVs durch das Digital-TV oder der Schallplatte durch die CD führte nicht zum Entstehen eines neuen Gattungsbegriffs. ${ }^{25}$ Das gleiche gilt für Innovationen, die allein die Ebene der Formate und Genres innerhalb einer Mediengattung betrafen, beispielsweise den Tonfilm, das Hörbuch oder die Tabloid-Zeitung. Der Begriff der „neuen Medien“ wird damit immer nur für solche medientechnischen Innovationen verwendet, die sowohl das Potenzial für neue inhaltliche Gestaltungsmöglichkeiten als auch das Potenzial für zusätzliche Rezeptionsmöglichkeiten bieten. Wenn neue Mediengattungen aber v. a. über eine Ausweitung des medialen Angebotes bzw. über neue Nutzungsmöglichkeiten definiert werden, ist es zunächst nicht verwunderlich, wenn ihre Substitutionswirkungen auf das jeweils existierende Angebot gering sind.

Alle hier präsentierten Ergebnisse unterliegen - neben der eingangs diskutierten Problematik der Abgrenzung einer „Mediengattung Internet“ - weiteren methodischen Einschränkungen. So werden für die ARD/ZDF-Langzeitstudie Massenkommunikation zu verschiedenen Zeitpunkten unterschiedliche Personen befragt und ihre Zeitverwendung wird dabei mittels Tagesablaufinterview jeweils nur für einen Tag erhoben. Die Daten erlauben deshalb auf Individualebene keine Aussagen über die Stärke langfristiger oder kurzfristiger Substitutionsbeziehungen. Nur für die Gesamtheit aller Befragten oder für einzelne Teilgruppen lässt sich überprüfen, ob eine höhere Nutzungsdauer oder ein höherer Zeitanteil (d. h. eine stärkere Präferenz) einer Mediengattung „im Durchschnitt" mit einer niedrigeren Nutzungsdauer oder einem niedrigeren Zeitanteil (d. h. einer schwächeren Präferenz) für ein anderes Medium einhergeht. Auch bei der Operationalisierung des Konstruktes Medienpräferenz muss unterstellt werden, dass sich aufgrund einer annähernd gleichmäßigen Verteilung der Tagesablaufinterviews über alle Wochentage die situativ bedingten Abweichungen der einzelnen Individuen von ihrer durchschnittlichen Zeitverwendung im Aggregat ausgleichen. Schließlich erfolgt die Abgrenzung zwischen den Zeitarten Reproduktionszeit und Freizeit auf Grundlage der in den Tagesablaufinterviews erhobenen allgemeinen Tätigkeiten. In der Zeitart Freizeit sind deshalb auch Tätigkeiten mit Verpflichtungscharakter im familiären und sozialen Umfeld enthalten, die sich im Hinblick auf die Möglichkeiten einer aufmerksamen Mediennutzung nicht von der Reproduktionszeit unterscheiden.

Für differenzierte Modellschätzungen auf Grundlage eines mikroökonomisch fundierten Modells der Mediennutzung als Zeitallokation sollten deshalb die Datengrundlagen in dreifacher Hinsicht verbessert werden:

1. Eine Erhebung der Zeitverwendung über einen längeren Zeitraum - im Idealfall für eine „künstliche Woche“ - ermöglicht es, die Einflüsse verfügbarer Zeitmengen auf die Mediennutzung über ein verbessertes Messmodell der Medienpräferenzen präziser zu erfassen. Hier könnte die Tagebuchmethodik den damit verbundenen Erhebungsaufwand in Grenzen halten.

2. Eine differenzierte Erfassung der allgemeinen Tätigkeiten, wie sie beispielsweise in den Zeitbudgetstudien des Statistischen Bundesamtes erfolgt, würde die Abgrenzung der Zeitarten Freizeit und Reproduktionszeit verbessern. Damit könnten die Unterschiede in der Wirkung von Zeitrestriktionen auf die Mediennutzung in Zeiträumen mit unterschiedlich hoher Disponibilität noch genauer analysiert werden.

3. Schließlich ist eine differenziertere Erfassung der jeweils genutzten Medieninhalte wünschenswert, die es erlaubt, auch die Substitutionsbeziehungen auf Genreebene

25 In Arbeiten aus den USA werden allerdings in der Regel broadcasting TV und cable TV als zwei unterschiedliche Mediengattungen betrachtet, da sie sich in ihrer Angebotsbreite und in ihrer Finanzstruktur stark unterscheiden (vgl. Dimmick 1993). 
(insbesondere zwischen Informations- und Unterhaltungsangeboten) genauer $\mathrm{zu}$ untersuchen. Es ist zu vermuten, dass sich dort stärkere Substitutionsbeziehungen zeigen als auf der Ebene der Mediengattungen.

\section{Literatur}

Becker, G. S. (1962): Irrational Behaviour and Economic Theory. In: Journal of Political Economy, 70 (1), S. 1-13. Deutsch in: Becker, G. S. (1993), Der ökonomische Ansatz zur Erklärung menschlichen Verhaltens. Tübingen: J. C. B. Mohr, S. 167-186.

Becker, G. S. (1965): A Theory of the Allocation of Time. In: Economic Journal, 75, S. 493-517. Deutsch in: Becker, G. S. (1993), Der ökonomische Ansatz zur Erklärung menschlichen Verhaltens. Tübingen: J. C. B. Mohr, S. 97-130.

Blumler, J./Katz, E. (Hg.) (1974): The Uses of Mass Communications. Current Perspectives in Gratifications Research. Beverly Hills: Sage.

Bromley, R./Bowles, D. (1995): Impact of Internet use on traditional news media. In: Nerwspaper Research Journal, 16(2), S. 14-27.

De Waal, E./Schönbach, K./Lauf, E. (2005): Online newspapers: A substitute or complement for print newspapers and other information channels? In: Communications 30(1), S. 55-72.

Dimmick, J. W. (1993): Ecology, Economics, and gratification utilities. In: Alexander, A./Owers, J./Carveth, R. (Hg.), Media economics: Theory and practice. Hillsdale, NJ: Laurence Erlbaum, S. 135-156.

Dimmick, J. W. (2003): Media Competition and Media Coexistence. The Theory of the Niche. Mahwah, NJ: Routledge.

Dimmick, J. W./Chen, Y./Li, Z. (2004): Competition between the Internet and Traditional News Media: The Gratification-Opportunities Niche Dimension. In: Journal of Media Economics, 17(1), S. 19-33.

Ehling, M./Holz, E./Kahle, I. (2001): Erhebungsdesign der Zeitbudgeterhebung 2001/2002. In: Wirtschaft und Statistik, 6/2001, S. 427-436.

Frey, B. S. (2004): Inspirierende Ökonomie und die Medien. In: Siegert, G./Lobigs, F. (Hg.), Zwischen Marktversagen und Medienvielfalt. Medienmärkte im Fokus newer medienökonomischer Anwendungen. Baden-Baden: Nomos, S. 31-36.

Haas, A. (2007): Medienmenüs. Der Zusammenhang zwischen Mediennutzung, Sinus-Milieus und Soziodemographie. München: Fischer.

Hagen, L. (1998): Online-Nutzung und Nutzung von Massenmedien. In: Rössler, P. (Hg.), Onlinekommunikation: Beiträge zur Nutzung und Wirkung. Opladen: Westdeutscher, S. 105-122.

Hasebrink, U./Popp, J. (2006): Media Repertoires as a Result of Selective Media Use. A Conceptual Approach to the Analysis of Patterns of Exposure. In: Communications, 31(2), S. 369-387.

Hasebrink, U./Domeyer, H. (2012): Media Repertoires as Patterns of Behaviour and as Meaningful Practices. A Multimethod Approach to Media Use in Converging Media Environments. In: Participations. Journal of Audience E Reception Studies, 9(2), S. 757-779.

Homburg, C./Klarmann, M./Pflesser, C. (2008a): Konfirmatorische Faktorenanalyse. In: Herrmann, A./Homburg, C./Klarmann, M. (Hg.), Handbuch Marktforschung. Methoden - Anwendungen - Praxisbeispiele. Wiesbaden: Gabler, S. 271-303.

Homburg, C./Pflesser, C./Klarmann, M. (2008b): Strukturgleichungsmodelle mit latenten Variablen: Kausalanalyse. In: Herrmann, A./Homburg, C./Klarmann, M. (Hg.), Handbuch Marktforschung. Methoden - Anwendungen - Praxisbeispiele. Wiesbaden: Gabler, S. 547-577.

Jäckel, M. (1992): Mediennutzung als Niedrigkostensituation. Anmerkungen zum Nutzen- und Belohnen-Ansatz. In: Medienpsychologie, 4(4), S. 246-266.

Kiefer, M.-L. (1989): Medienkomplementarität und Medienkonkurrenz. Notizen zum weitgehend ungeklärten „Wettbewerbsverhältnis“ der Medien. In: Kaase, M./Schulz, W. (Hg.), Massenkommunikation Theorien, Methoden, Befunde. Opladen: Westdeutscher. Sonderheft der Kölner Zeitschrift für Soziologie und Sozialpsychologie, Nr. 30, S. 337-350.

Kiefer, M-L. (2005): Medienökonomik. 2. Auflage. München u. a.: R. Oldenbourg. 
Fritz, I./Klingler, W. (2003): Zeitbudgets und Tagesablaufverhalten in Deutschland: Die Position der Massenmedien. Ergebnisse auf Basis der ARD/ZDF-Studie Massenkommunikation 2000. In: Media Perspektiven, Heft 1, S. 12-23.

Kolo, C./Meyer-Lucht, R. (2007): Erosion der Intensivleserschaft: eine Zeitreihenanalyse zum Konkurrenzverhältnis von Tageszeitungen und Nachrichtensites. In: Medien E Kommunikationswissenschaft, 55(4), S. 513-533.

Kroeber-Riel, W./Weinberg, P./Gröppel-Klein, A. (2011): Konsumentenverhalten. 9. Auflage. München: Vahlen.

Langenbucher, W. R. (1968): Das Fernsehen und die Presse. Ein Beitrag zur Frage der journalistischen Konkurrenz oder Ergänzung. In: Rundfunk und Fernsehen, 16, S. 1-12.

Lazarsfeld, P. F./Kendall, P. F. (1948): Radio Listening in America. The People look at RadioAgain. New York: Prentice-Hall.

Lerg, W. B. (1981): Verdrängen oder ergänzen die Medien einander? Innovationen und Wandel in Kommunikationssystemen. In: Publizistik, 26(2), S. 193-201.

Lüdtke, H. (1984): Gleichförmigkeiten im alltäglichen Freizeitverhalten. In: Zeitschrift für Soziologie 13, S. 346-362.

Markus, A. (2007): Media Diversity and Substitutability: Problems with the FCC's Diversity Index In: I/S: A Journal of Law and Policy for the Information Society, 3(1), S. 83-115.

Meulemann, H. (2012): Die psychischen Kosten von Freizeitaktivitäten und die Entfaltungschancen des Fernsehangebotes. Warum das Fernsehen in Deutschland auch nach dem Aufkommen des Internets die beliebteste Freizeitaktivität bleibt. In: Medien $\mathcal{E}$ Kommunikationswissenschaft, 60(2), S. 240-261.

Mögerle, U. (2009): Substitution oder Komplementarität? Die Nutzung von Online- und PrintZeitungen im Wandel. Konstanz: UVK.

Oehmichen, E./Schröter, C. (2007): Zur typologischen Struktur medienübergreifender Nutzungsmuster. In: Media Perspektiven, 8/2007, S. 406-421.

Opaschowski, H. W. (2008): Einführung in die Freizeitwissenschaft. 5. Auflage. Wiesbaden: VS.

Owen, B. M./Beebe, J. /Manning, W. (1974): Television economics. Lexington, MA: D. C. Heath.

Owen, B. M./Wildman, S. S. (1992): Video economics. Cambridge, MA: Harvard University Press.

Peiser, W. (2002): Wolfgang Riepl. In: Holtz-Bacha, C./Kutsch, A. (Hg.), Schlüsselwerke für die Kommunikationswissenschaft. Opladen: Westdeutscher, S. 368-373.

Picard, R. G. (1989): Media Economics. Concepts and Issues. Newbury Park: Sage.

Reitze, H./Ridder, C.-M. (Hg.) (2011): Massenkommunikation VIII. Eine Langzeitstudie zur Mediennutzung und Medienbewertung 1964-2010. Baden-Baden: Nomos.

Ridder, C.-M./Engel, B. (2010): Massenkommunikation 2010: Mediennutzung im Intermediavergleich. In: Media Perspektiven, 11/2010, S. 523-536.

Ridder, C.-M./Turecek, I. (2011): Medienzeitbudgets im Tagesablaufverhalten. In: Media Perspektiven, 12/2011, S. 570-582.

Riepl, W. (1987): Das Nachrichtenwesen des Altertums mit besonderer Rücksicht auf die Römer. Berlin/Leipzig 1913. Abgedruckt unter dem Titel „Das Gesetz von der Komplementarität“ In: Brobowski, M./Duchkowitsch, W./Haas, H./Langenbucher, W. R. (Hg.), Medien- und Kommunikationsgeschichte. Ein Textbuch zur Einführung. Wien: Braunmüller, S. 144-148.

Rosengren, K. E./Wenner, L. A./Palmgreen, P. (Hg.) (1985): Media gratifications research: Current perspectives. Beverly Hills: Sage.

Salwen, M. B./Garrison, B./Driscoll P. D. (2004): Online News and the Public. Mahwah, NJ: Routledge.

Scherer, H./Schlütz, D. (2004): Das neue Medien-Menü: Fernsehen und WWW als funktionale Alternativen? In: Publizistik, 49, S. 6-24.

Scheuch, E. K. (1972): Die Problematik der Freizeit in der Massengesellschaft. In: Scheuch, E. K./ Meyersohn, R. (Hg.), Soziologie der Freizeit. Köln: Kiepenheuer und Witsch, S. 23-44.

Seufert, W./Ehrenberg, M. (2007): Microeconomic Consumption. Theory and Individual Media Use: Empirical Evidence from Germany. In: Journal of Media Business Studies, 4(3), S. 21-39.

Seufert, W./Gundlach, H. (2012): Medienregulierung in Deutschland. Ziele - Konzepte - Maßnahmen. Baden-Baden: Nomos. 
Seufert, W./Wilhelm, C. (2013): Mediennutzung als Zeitallokation. Zum Einfluss der verfügbaren Zeit auf die Medienauswabl. Baden-Baden: Nomos. (Druck in Vorbereitung)

Simon, H. A. (1959): Theories of decision making in economics and behavioral science. In: American Economic Review, 49(3), S. 253-283.

Steiner, P. O. (1952): Program patterns and preferences and the workability of competition in radio broadcasting. In: Quarterly Journal of Economics, 66, S. 194-223.

Van Eimeren. B./ Frees, B. (2012): 76 Prozent der Deutschen online - neue Nutzungssituationen durch mobile Endgeräte. Ergebnisse der ARD/ZDF-Onlinestudie 2012. In: Media Perspektiven, Heft 7-8, S. 362-379.

Varian, H. R. (2007): Grundzüge der Mikroökonomik. 7. Auflage. München: Oldenbourg.

Waldfogel, J. (2002): Consumer Substitution among Media. Federal Communications Commission (FCC) Media Bureau Staff Research Paper 2002-3 (Sept 2002), http://transition.fcc.gov/own ership/materials/already-released/consumer090002.pdf [15.08.2013].

Waterman, D. (2006): The Economics of Media Programming. In: Albarran, A. B./Chan-Olmsted, S./Wirth, M. O. (Hg.), Handbook of Media Management and Economics. Mahwah, NJ: Lawrence Erlbaum Associates, S. 386-416.

Weiber, R./Mühlhaus, D. (2010): Strukturgleichungsmodellierung. Eine anwendungsorientierte Einfübrung in die Kausalanalyse mit Hilfe von AMOS, SmartPLS und SPSS. Berlin, Heidelberg: Springer.

Wilhelm, C./Seufert, W. (2011): Mediennutzung und Zeitverfügbarkeit. In: Suckfüll, M./Schramm, H./Wünsch, C. (Hg.), Rezeption und Wirkung in zeitlicher Perspektive. Baden-Baden: Nomos, S. $111-128$

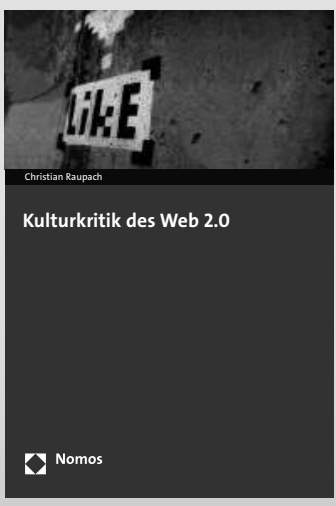

\section{Kulturkritik des Web 2.0}

Von Christian Raupach

2013, 239 S., brosch., 44,- $€$

ISBN 978-3-8487-0636-5

Web 2.0 macht Kulturproduktion einfacher, aber nicht freier. Christian Raupachs „Kulturkritik des Web 2.0" argumentiert mithilfe der Kulturindustrie-These, dass Kulturproduktion nach wie vor bestimmt ist von Warenförmigkeit und manipulierten Bedürfnissen.

Bestellen Sie jetzt telefonisch unter 07221/2104-37.

Portofreie Buch-Bestellungen unter www.nomos-shop.de/21256

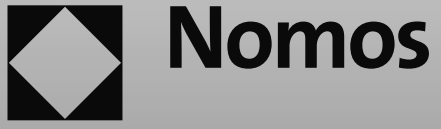


Anhang

Tabelle 3: Globale Fitmaße der Mebrgruppenkausalanalysen zu langfristigen Substitutionseffekten

\begin{tabular}{lccccc}
\hline Modell & CMIN/DF & RMSEA & SRMR & TLI & CFI \\
\hline Freizeit & & & & & \\
\hline TV-Nutzung & 20,93 & 0,044 & 0,083 & 0,97 & 0,97 \\
Radionutzung & 28,90 & 0,052 & 0,037 & 0,96 & 0,96 \\
Internetnutzung* & 64,79 & 0,078 & 0,032 & 0,90 & 0,90 \\
Zeitungsnutzung & 42,53 & 0,063 & 0,063 & 0,94 & 0,94 \\
nicht-mediale Aktivitäten & 20,06 & 0,043 & 0,0832 & 0,96 & 0,96 \\
\hline Reproduktionszeit & & & & & \\
\hline TV-Nutzung & 25,00 & 0,048 & 0,032 & 0,97 & 0,97 \\
Radionutzung & 21,40 & 0,044 & 0,039 & 0,97 & 0,97 \\
Zeitungsnutzung & 25,24 & 0,048 & 0,057 & 0,97 & 0,97 \\
\hline
\end{tabular}

*1995: PC-Nutzung

Quelle: Eigene Berechnung auf Basis der ARD/ZDF-Langzeitstudie Massenkommunikation; Befragte mit Freizeit Mo-Fr (1995: N = 4100; 2005: N = 3201; 2010: N = 3172); Befragte mit Reproduktionszeit Mo-Fr (1995: $\mathrm{N}=4207,2005: \mathrm{N}=3222,2010: \mathrm{N}=3199)$. Methode: Maximum Likelihood; Modell: Measurement Intercepts.

Tabelle 4: Globale Fitmaße der Modelle zu kurzfristigen Substitutionsbeziebungen 2005

\begin{tabular}{|c|c|c|c|c|c|}
\hline Modell & CMIN/DF & RMSEA & SRMR & TLI & $\mathrm{CFI}$ \\
\hline TV- und Radionutzung & 17,04 & 0,071 & 0,055 & 0,96 & 0,97 \\
\hline TV- und Internetnutzung & 17,93 & 0,073 & 0,055 & 0,96 & 0,97 \\
\hline TV- und Zeitungsnutzung & 19,52 & 0,076 & 0,059 & 0,95 & 0,96 \\
\hline $\begin{array}{l}\text { TV-Nutzung und nicht-mediale Freizeitak- } \\
\text { tivitäten }\end{array}$ & 22,25 & 0,081 & 0,076 & 0,93 & 0,94 \\
\hline Radio- und Zeitungsnutzung & 15,22 & 0,067 & 0,036 & 0,96 & 0,97 \\
\hline $\begin{array}{l}\text { Radionutzung und nicht-mediale Freizeit- } \\
\text { aktivitäten }\end{array}$ & 13,36 & 0,062 & 0,064 & 0,96 & 0,97 \\
\hline Internet- und Zeitungsnutzung & 12,32 & 0,059 & 0,037 & 0,97 & 0,98 \\
\hline $\begin{array}{l}\text { Internetnutzung und nicht-mediale Frei- } \\
\text { zeitaktivitäten }\end{array}$ & 12,81 & 0,061 & 0,062 & 0,96 & 0,97 \\
\hline $\begin{array}{l}\text { Zeitungsnutzung und nicht-mediale Frei- } \\
\text { zeitaktivitäten }\end{array}$ & 16,54 & 0,070 & 0,066 & 0,95 & 0,96 \\
\hline
\end{tabular}

Quelle: Eigene Berechnung auf Basis LMK 2005, Befragte mit Freizeit, Mo-Fr (n=3201). Methode: Maximum Likelihood 
Tabelle 5: Globale Fitmaße der Modelle zu kurzfristigen Substitutionsbeziehungen 2010

\begin{tabular}{lccccc}
\hline Modell & CMIN/DF & RMSEA & SRMR & TLI & CFI \\
\hline TV- und Radionutzung & 16,23 & 0,069 & 0,044 & 0,96 & 0,97 \\
TV- und Internetnutzung & 17,29 & 0,072 & 0,041 & 0,96 & 0,97 \\
TV- und Zeitungsnutzung & 17,65 & 0,072 & 0,035 & 0,96 & 0,97 \\
TV-Nutzung und nicht-mediale Aktivitäten & 21,56 & 0,080 & 0,087 & 0,93 & 0,93 \\
Radio- und Internetnutzung & 13,57 & 0,063 & 0,044 & 0,97 & 0,98 \\
Radio- und Zeitungsnutzung & 11,97 & 0,059 & 0,040 & 0,97 & 0,98 \\
Radionutzung und nicht-mediale Aktivitä- & 14,96 & 0,066 & 0,069 & 0,95 & 0,96 \\
ten & 8,13 & 0,047 & 0,031 & 0,98 & 0,99 \\
Internet- und Zeitungsnutzung & 14,33 & 0,065 & 0,070 & 0,96 & 0,96 \\
Internetnutzung und nicht-mediale Aktivi- & & & & & \\
täten & 17,58 & 0,072 & 0,070 & 0,94 & 0,95 \\
Zeitungsnutzung und nicht-mediale Aktivi- & & & & & \\
täten & & &
\end{tabular}

Quelle: Eigene Berechnung auf Basis LMK 2010, Befragte mit Freizeit, Mo-Fr ( $\mathrm{n}=3172)$. Methode: Maximum Likelihood

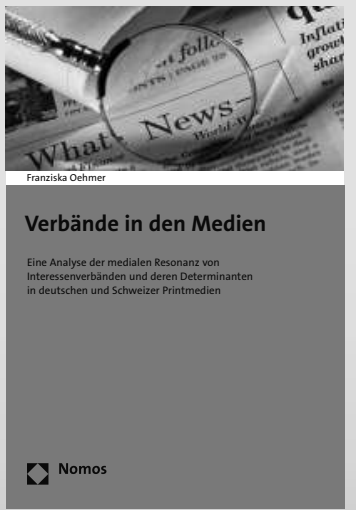

\section{Verbände in den Medien}

Eine Analyse der medialen Resonanz von Interessenverbänden und deren Determinanten in deutschen und Schweizer Printmedien

Von Franziska Oehmer 2013, 203 S., brosch., 34,- $€$ ISBN 978-3-8487-0494-1

Bestellen Sie jetzt telefonisch unter 07221/2104-37.

Portofreie Buch-Bestellungen unter www.nomos-shop.de/20964

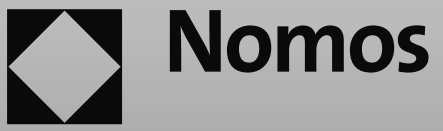

